Research paper

\title{
Homogenization accounting for size effects in particulate composites due to general interfaces
}

\author{
Soheil Firooz ${ }^{\mathrm{a}}$, George Chatzigeorgiou ${ }^{\mathrm{b}}$, Fodil Meraghni ${ }^{\mathrm{b}}$, Ali Javili ${ }^{\text {*a }}$ \\ ${ }^{a}$ Department of Mechanical Engineering, Bilkent University, Ankara 06800, Turkey \\ ${ }^{\mathrm{b}}$ LEM3-UMR 7239 CNRS, Arts et Metiers ParisTech Metz, 4 Rue Augustin Fresnel Metz 57078, France
}

\section{A R T I C L E I N F O}

\section{Keywords:}

Homogenization

Imperfect interface

Size effects

Particulate composites

Mori-Tanaka method

\begin{abstract}
A B S T R A C T
Two analytical approaches are developed to determine the overall size-dependent response of composites embedding general interfaces. The first approach extends the composite sphere assemblage (CSA) approach and the generalized self-consistent method (GSCM) to account for the general interface model resulting in new bounds and estimates on the macroscopic properties of particulate composites. In the second approach, we develop an interface-enhanced Mori-Tanaka method that not only determines the effective properties but also provides the state of the stress and strain in each phase of the medium. The general interface model captures both elastic and cohesive interface models. Computational analysis is carried out using the finite element method to verify the analytical results. A remarkable agreement between the proposed analytical solutions and the computational results is obtained. A thorough parametric study is carried out to shed light on the role of the general interfaces in the overall behavior of composites. Motivated by the numerical and analytical findings, the material behavior is found to be bounded. Thus, two notions of ultimate bounds and size-dependent bounds are introduced and discussed.
\end{abstract}

\section{Introduction}

Almost all materials possess heterogeneous structures at certain length-scales. The overall behavior of heterogeneous materials can be determined from the response of their underlying micro-structures via the homogenization method pioneered by Hill (1963, 1972) and Ogden (1974). The classical homogenization method allows us to determine the overall behavior of composites based on the volume fraction, shape, orientation and distribution of their constituents. But classical homogenization cannot capture the size effects. As the characteristic length of a heterogeneous structure decreases e.g. in nanocomposites, the effects of the surface and interface energy on the overall material response increases (Brisard et al., 2010; Chatzigeorgiou et al., 2015; Duan and Karihaloo, 2007; Duan et al., 2005; Mogilevskaya et al., 2008; Nazarenko et al., 2017; Sharma, 2004; Sharma and Wheeler, 2007; Tian and Rajapakse, 2007; Fritzen and Leuschner, 2015; Javili et al., 2013a; Monteiro et al., 2011; Yvonnet et al., 2008). Comparisons with experiments and atomistic simulations in (Davydov et al., 2013; Elsner et al., 2017; He and Lilley, 2008; Levitas and Samani, 2011; Olsson and Park, 2012; Park and Klein, 2008) justify that the size effects due to interfaces are physically meaningful. Emerging applications of nano-composites demand for better understanding of the interfacial effects on the overall response of heterogeneous media. In this contribution, we present two novel analytical methods to determine the overall behavior of particulate composites via a homogenization framework accounting for general interfaces. Furthermore, a computational analysis is carried out using the finite element method to verify the proposed analytical solutions. A two-dimensional counterpart of this work associated with fiber composites is only very recently published by Firooz et al. (2019a).

The term general interface here refers to a zero-thickness model allowing for both displacement and traction discontinuities across the interface (Sanchez-Palencia, 1970; Pham Huy and Sanchez-Palencia, 1974; Hashin, 2001; Javili, 2017). The bonding between the constituents of composites is important and can significantly influence the overall material response. The assumption of perfect bonding at interfaces is, in general, inadequate to describe the physical nature and mechanical behavior of the interface region and therefore, in many cases, an imperfect interface model should be employed. Fig. 1 categorizes the main interface models based on the continuity of the displacement or traction fields across the interface. The interface is referred to as perfect if both the displacement and traction jumps are

\footnotetext{
* Corresponding author.

E-mail addresses: soheil.firooz@bilkent.edu.tr (S. Firooz), georges.chatzigeorgiou@ensam.eu (G. Chatzigeorgiou), fodil.meraghni@ensam.eu (F. Meraghni), ajavili@bilkent.edu.tr (A. Javili).
} 


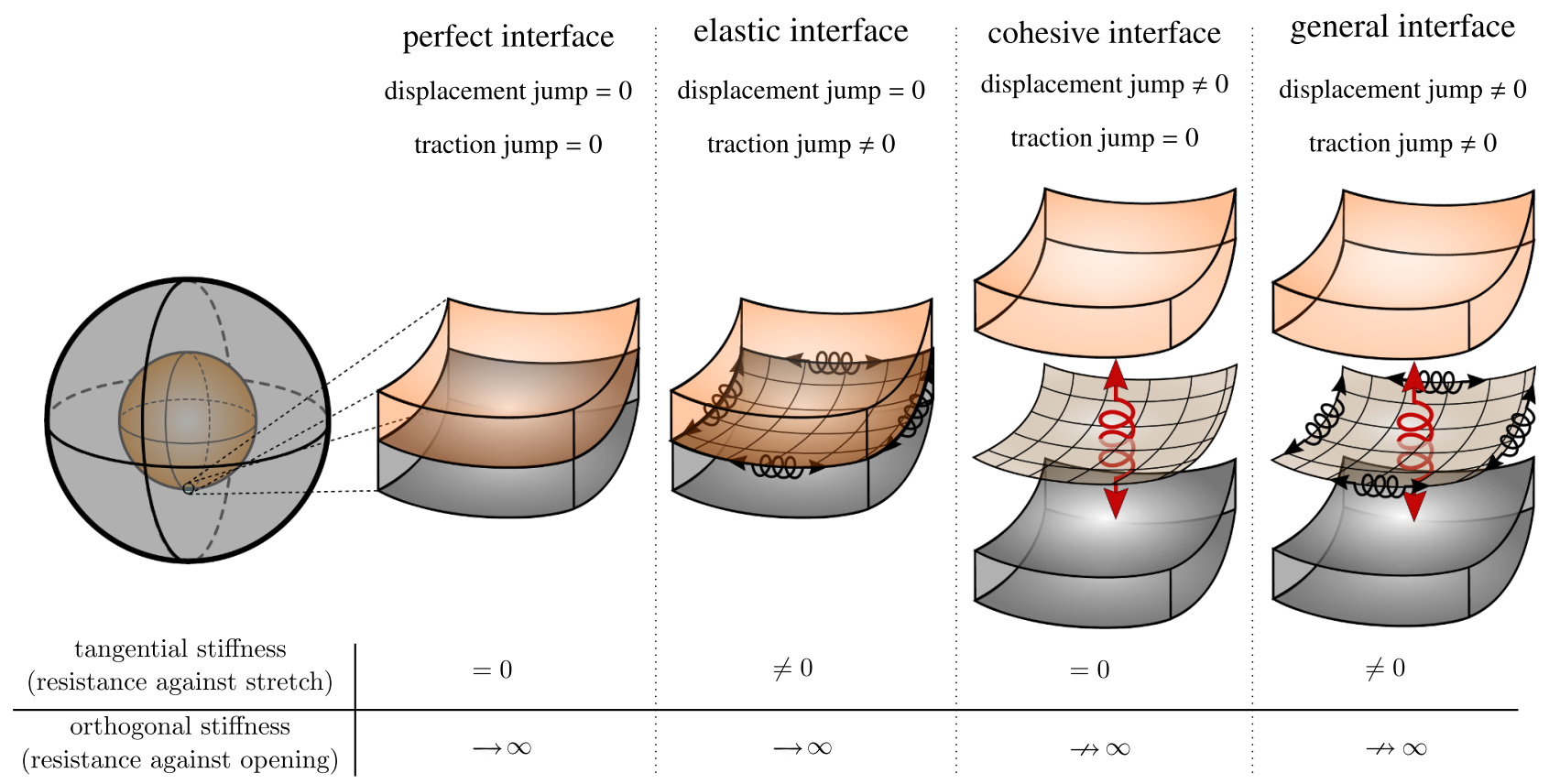

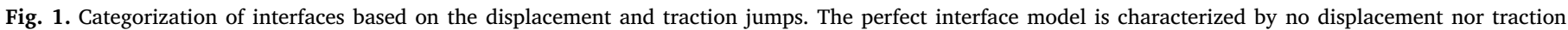

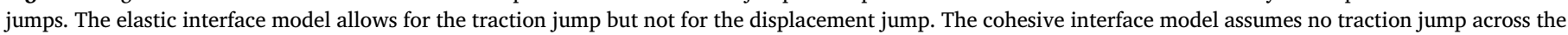

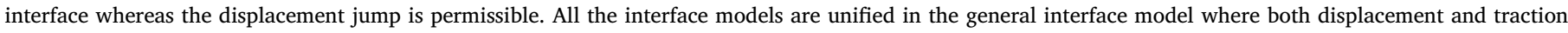
jumps are permissible.

zero. The elastic interface model allows for the traction jump whereas the displacement jump vanishes. The traction jump across the elastic interface is the result of the stress divergence along the interface conforming to a generalized Young-Laplace equation (Chen et al., 2006; Javili et al., 2013c; Javili, 2018). Interface elasticity theory spans a variety of studies from the fundamental works (Gurtin and Murdoch, 1975; Moeckel, 1975; Murdoch, 1976; Daher and Maugin, 1986; dell'Isola and Romano, 1987; Fried and Gurtin, 2007; Klarbring, 1991) and has been further investigated in (Huang and Sun, 2007; Huang and Wang, 2006; Altenbach and Eremeyev, 2011; Chhapadia et al., 2011; Cordero et al., 2016; Dingreville et al., 2014; Dingreville and Qu, 2008; Duan et al., 2009; Fried and Todres, 2005; Javili et al., 2013b; Liu et al., 2017; Steigmann and Ogden, 1999; Wang et al., 2010; Zhong and Meguid, 1997; Fedotov, 2018), among others. In the cohesive interface model the traction field is continuous across the interface unlike the displacement field. The cohesive interface model dates back to the seminal works (Barenblatt, 1959; Dugdale, 1960; Barenblatt, 1962) and has been extensively studied in (Needleman, 1987; van den Bosch et al., 2006; Wells and Sluys, 2001; Remmers et al., 2008; Moës and Belytschko, 2002; Alfano and Crisfield, 2001; Despringre et al., 2016; Dimitri et al., 2015; Fagerström and Larsson, 2006; Gasser and Holzapfel, 2003; Ortiz and Pandolfi, 1999; Park and Paulino, 2011; Park et al., 2009; Wu et al., 2016; Qian et al., 2017; Wang et al., 2005; Liu et al., 2019; Brassart et al., 2009) from both analytical and computational aspects. The general interface model unifies all the aforementioned models allowing for both traction and displacement jumps. The general interface model has been examined in a seminal work of Hashin (2002) and further studied in (Benveniste and Miloh, 2001; Benveniste, 2006; Gu et al., 2011; Monchiet and Bonnet, 2010; Chatzigeorgiou et al., 2017; Gu et al., 2014; Koutsawa et al., 2018; Firooz and Javili, 2019) among others.

The term size here refers to the physical size of the micro-structure. The definition of the size is schematically illustrated in Fig. 2. The radii of the inclusion and the matrix can be calculated for a given volume fraction $f$ and size $\ell$. Throughout the manuscript, the macroscopic quantities are distinguished from their microscopic counterparts by a left superscript " $\mathrm{M}$ ". That is, $\left.\mathbf{M}_{\bullet} \cdot\right\}$ is a quantity at the macro-scale with its microscopic counterpart being $\{\cdot\}$. Moreover, quantities referring to the interface are distinct from the bulk quantities by a bar placed on top them. For instance, $\{\hat{\theta}\}$ refers to an interface quantity with its bulk counterpart $\{\cdot\}$. The average and the jump of a quantity $\{\cdot\}$ across the interface are denoted by $\{\{\{\bullet\}\}\}$ and $[[\{\bullet\}]]$, respectively.

The rest of the manuscript is organized as follows. Section 2 provides a brief discussion on the governing equations and problem definition. The analytical approaches are presented in Section 3. First the preliminaries of the RVE problem for particulate composites are introduced briefly. Next, the generalized self-consistent method and composite sphere assemblage method are extended to account for interfaces. This is then followed by developing the new interface-enhanced Mori-Tanaka approach to incorporate interface effects in particulate composites using the modified Eshelby's heterogeneity problem. In Section 4, the analytical and computational solutions are compared through a set of numerical examples. A thorough comparison between our methodology and available analytical estimates in the literature is provided in Section 5. Section 6 concludes our work and provides further outlooks for future studies.

\section{Governing equations}

The governing equations of a continua embedding a general interface are briefly discussed in this section. Note that for the sake of brevity, only the final form of the most essential equations are stated. Detailed derivations are available in (Chatzigeorgiou et al., 2017; Javili, 2017; Javili et al., 2017). Consider a continuum body that occupies the configuration ${ }^{\mathrm{M}_{\mathcal{B}}}$ at the macro-scale corresponding to a heterogeneous medium, as shown in Fig. 3, with its underlying simplified representative volume element (RVE) at the micro-scale denoted as $\mathcal{B}$. In a computational homogenization framework, a proper RVE must be chosen such that (i) it is large enough to contain adequate details about the micro-structure and (ii) it is small enough to guarantee the scale separation (Khisaeva and Ostoja-Starzewski, 2006; Temizer and Zohdi, 2007; Gitman et al., 2005). In analytical homogenization, the RVE is often replaced by a spherical micro-structure suitable to represent isotropic particulate composites. At the micro-scale, the constitutive 


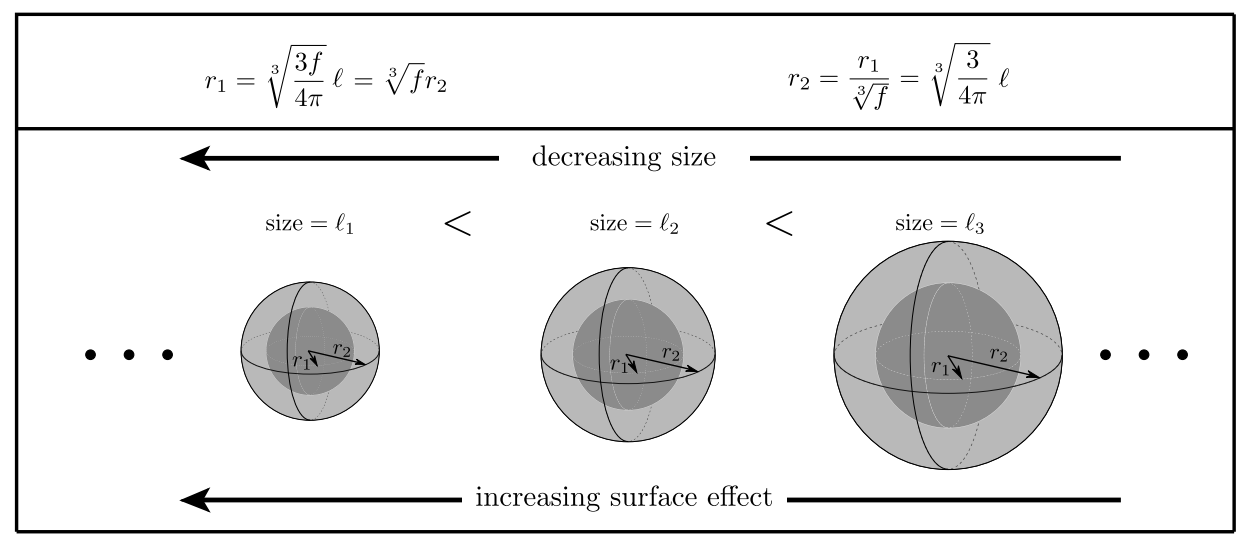

Fig. 2. Illustration of the term "size". Using the volume fraction, the radii of the inclusion and the matrix can be obtained for each specific size. As a result, size is proportional to the radius of the inclusion or that of the matrix.

behavior of each phase is assumed to be known and the overall macroscopic material response is obtained via solving the associated boundary value problem and proper averaging over the RVE (Saeb et al., 2016; Kanouté et al., 2009; Charalambakis et al., 2018; Matouš et al., 2017; Pindera et al., 2009). As shown in Fig. 3, we approximate the finite-thickness interphase between the constituents by the zerothickness general interface model. The interface $\mathcal{I}$, splits the microstructure into two disjoint subdomains $\mathcal{B}^{+}$and $\mathcal{B}^{-}$associated with the plus and minus sides of the interface, respectively. The unit normals to the external boundary and to the interface are denoted as $\boldsymbol{n}$ and $\overline{\boldsymbol{n}}$, respectively. Note, the interface unit normal points from the minus side of the interface to its plus side. Let $\boldsymbol{u}$ denote the displacement field in the bulk. The interface displacement $\overline{\boldsymbol{u}}$ is defined by the average displacement across the interface $\{\{\boldsymbol{u}\}\}$. The displacement average and the displacement jump across the interface are defined by

$\{\boldsymbol{u}\}:=\frac{1}{2}\left[\boldsymbol{u}^{+}+\boldsymbol{u}^{-}\right] \quad$ and $\quad[[\boldsymbol{u}]]:=\boldsymbol{u}^{+}-\boldsymbol{u}^{-}$,

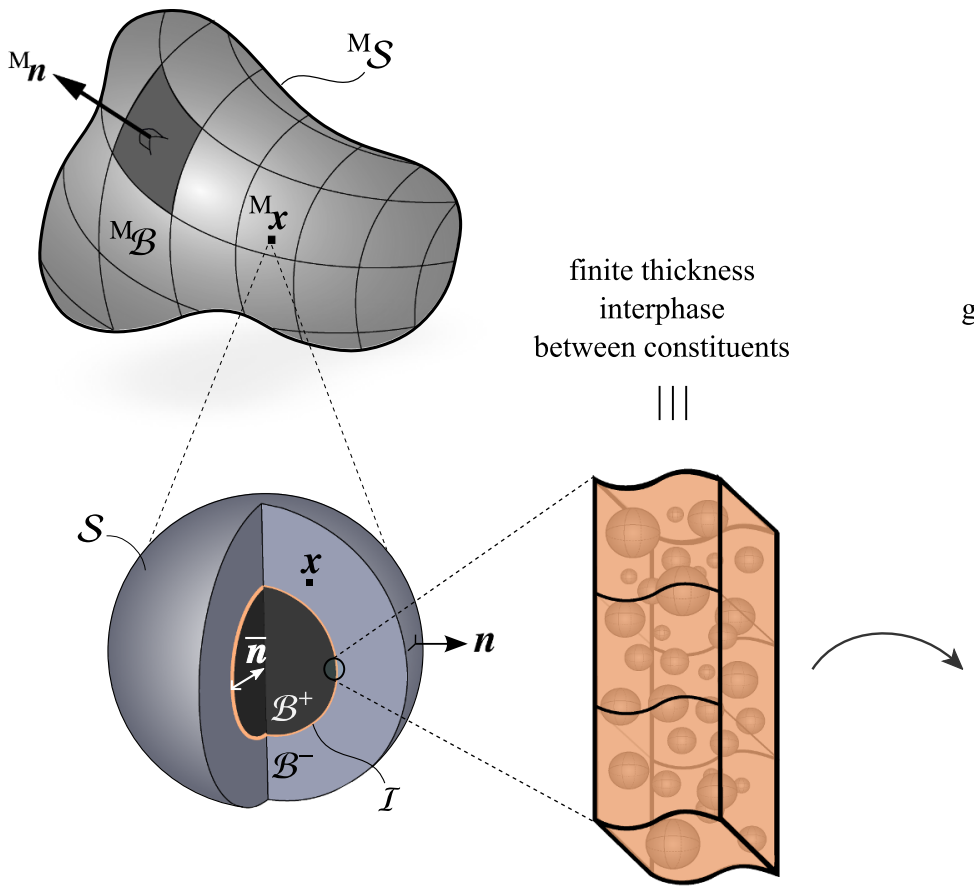

zero-thickness general interface model
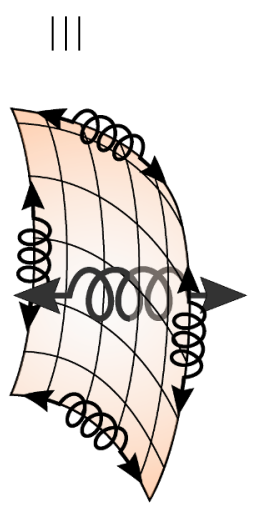

Fig. 3. Problem definition for homogenization including the general interface model. The macro-structure is shown with its underlying RVE. The constitutive laws at the micro-scale are assumed to be known and the macroscopic behavior is obtained via solving the boundary value problem at the micro-scale. A finitethickness interphase is replaced with a zerothickness interface model. The classical interface models cannot capture heterogeneous material layer and thus, the general interface model is required. 
interface free energy density depends on both the interface strain and interface displacement jump as $\bar{\psi}=\bar{\psi}(\overline{\boldsymbol{\varepsilon}},[[\boldsymbol{u}]])$. The contributions of the interface curvature or higher gradients of the interface strain are not taken into account in this study. The constitutive relations in the bulk and on the interface in terms of their free energy densities read

$\sigma:=\frac{\partial \psi}{\partial \boldsymbol{\varepsilon}} \quad$ in $\mathcal{B}, \quad \overline{\boldsymbol{\sigma}}:=\frac{\partial \bar{\psi}}{\partial \overline{\boldsymbol{\varepsilon}}} \quad$ and $\quad \overline{\boldsymbol{t}}:=\frac{\partial \bar{\psi}}{\partial \llbracket \boldsymbol{u} \rrbracket} \quad$ on $\mathcal{I}$,

with $\overline{\boldsymbol{t}}$ being the interface traction and $\overline{\boldsymbol{t}}=\{\{\sigma\}\} \cdot \overline{\boldsymbol{n}}$. In the absence of external forces, the balance equations in the bulk and on the interface read

$$
\begin{aligned}
& \text { bulk }\left\{\begin{array}{l}
\operatorname{div} \sigma=\boldsymbol{0} \text { in } \mathcal{B}, \\
\sigma \cdot \boldsymbol{n}=\boldsymbol{t} \text { on } \mathcal{S},
\end{array} \quad\right. \text { interface } \\
& \begin{cases}\overline{\operatorname{div}} \bar{\sigma}+\llbracket \sigma \rrbracket \cdot \overline{\boldsymbol{n}}=\mathbf{0} & \text { along } \mathcal{I}, \\
\{\{\sigma\} \cdot \overline{\boldsymbol{n}}=\overline{\boldsymbol{t}} & \text { across } \mathcal{I},\end{cases}
\end{aligned}
$$

where $t$ is the traction on the boundary $\mathcal{S}$. Note, the interface curvature operator is embedded within the interface divergence operator $\overline{\operatorname{div}}\{\bullet\}=\operatorname{grad}\{\bullet\}: \overline{\boldsymbol{i}}$. The bulk material response is assumed to be standard and isotropic elastic taking the form $\sigma=2 \mu \boldsymbol{\varepsilon}+\lambda[\boldsymbol{\varepsilon}: \boldsymbol{i}] \boldsymbol{i}$ where $\lambda$ and $\mu$ are the Lamé parameters. For the interface, we additively decompose the material response into an orthogonal response across the interface and a tangential response along the interface

$\overline{\boldsymbol{t}}=\bar{k}[[\boldsymbol{u}]] \quad$ and $\quad \overline{\boldsymbol{\sigma}}=2 \bar{\mu} \overline{\boldsymbol{\varepsilon}}+\bar{\lambda}[\overline{\boldsymbol{\varepsilon}}: \overline{\boldsymbol{i}}] \overline{\boldsymbol{i}}$,

respectively, with $\bar{\lambda}$ and $\bar{\mu}$ being the interface Lamé parameters representing the interface resistance against in-plane stretches and $\bar{k}$ being the interface orthogonal resistance against opening.

Remark. Usually, the interface parameters of zero-thickness interface models and the bulk parameters of an equivalent finite-thickness interphase are linked through the thickness of the interphase, see (Hashin, 1991; Wang et al., 2005; Duan et al., 2007; Gu et al., 2014; Chatzigeorgiou et al., 2015) for more details. The exact relations between these properties depend on the order of anisotropy and the type of imperfection of the interface model. As a general rule, the $\bar{\lambda}, \bar{\mu}$ are given by a combination of the equivalent interphase properties multiplied by the interphase thickness while $\bar{k}$ is proportional to the inverse of the thickness of the interphase.

Remark. While not presented here, our methodology is, in principle, extensible for nonlinear interface conditions. The relations (5) can be adopted for nonlinear mechanisms, by considering field dependent $\bar{k}, \bar{\mu}$ and $\bar{\lambda}$, as well as proper evolution laws and activation criteria. Of course, a linearized incremental numerical scheme may be required (for instance, a return mapping algorithm approach) for introducing debonding or softening at the interface. An even more complex problem arises when the bulk materials are also nonlinear. In such cases, the non-uniformity of the fields inside each phase causes inconsistency in the validity of the analytical solutions provided in this manuscript. Various techniques can be employed to overcome such issues but they exceed the scopes of the current article and shall be studied in a separate contribution.

Equipped with the constitutive laws and the governing equations, we proceed with the micro to macro transition. In the classical computational homogenization framework, macroscopic quantities are related to their microscopic counterparts through volume averaging over the RVE. For this study, the classical definitions must be extended to incorporate the interfaces. The macroscopic stress and strain fields can be defined as surface integrals over the RVE's boundary as

$$
\begin{aligned}
\mathrm{M}_{\boldsymbol{\varepsilon}} & =\frac{1}{\mathscr{V}} \int_{\mathcal{S}} \frac{1}{2}[\boldsymbol{u} \otimes \boldsymbol{n}+\boldsymbol{n} \otimes \boldsymbol{u}] \mathrm{d} A \quad \text { and } \quad \mathrm{M}_{\boldsymbol{\sigma}} \\
& =\frac{1}{\mathscr{V}} \int_{\mathcal{S}} \boldsymbol{t} \otimes \boldsymbol{x} \mathrm{d} A,
\end{aligned}
$$

that, using the extended divergence theorem Javili et al. (2017), simplify to

$$
\begin{aligned}
\mathrm{M}_{\boldsymbol{\varepsilon}}= & \frac{1}{\mathscr{V}} \int_{\mathcal{B}} \boldsymbol{\varepsilon} \mathrm{d} V \\
& +\frac{1}{\mathscr{V}} \int_{\bar{I}} \frac{1}{2}[[[\boldsymbol{u}]] \otimes \overline{\boldsymbol{n}}+\overline{\boldsymbol{n}} \otimes[[\boldsymbol{u}]]] \mathrm{d} A \quad \text { and } \mathrm{M}_{\boldsymbol{\sigma}} \\
= & \frac{1}{\mathscr{V}} \int_{\mathcal{B}} \sigma \mathrm{d} V+\frac{1}{\mathscr{V}} \int_{I} \bar{\sigma} \mathrm{d} A .
\end{aligned}
$$

Next, an incremental energy equivalence, also known as the Hill-Mandel condition, between the scales is imposed in an extended form to account for the interface. The interface-enhanced Hill-Mandel condition reads

$\delta^{\mathrm{M}} \psi \stackrel{!}{=} \frac{1}{\mathscr{V}} \int_{\mathcal{B}} \delta \psi \mathrm{d} V+\frac{1}{\mathscr{V}} \int_{I} \delta \bar{\psi} \mathrm{d} A$,

where $\stackrel{!}{=}$ indicates that the equality is a condition. Employing the Hill's lemma, the Hill-Mandel condition (8) can be written as the boundary integral

$\int_{\mathcal{S}}\left[\delta \boldsymbol{u}-\delta^{\mathrm{M}} \boldsymbol{\varepsilon} \cdot \boldsymbol{x}\right] \cdot\left[\boldsymbol{t}-\mathrm{M}_{\boldsymbol{\sigma}} \cdot \boldsymbol{n}\right] \mathrm{d} A \stackrel{!}{=} 0$,

identifying suitable boundary conditions. Among all the boundary conditions satisfying the Hill-Mandel condition, the linear displacement boundary condition (DBC) and constant traction boundary condition (TBC) are imposed here. This choice is necessary in order to have a meaningful comparison between the computational and analytical results. See Firooz et al. (2019b) for a comprehensive study on the effects of the boundary condition and the RVE type on the overall response of composites.

\section{Analytical estimates}

This section elaborates the two new analytical approaches that we develop here to determine the overall behavior of composites embedding general interfaces. The first approach is a direct extension of the composite sphere assemblage (CSA) approach and the generalized selfconsistent method (GSCM) which incorporates the general interfaces providing bounds and estimates on the macroscopic properties. The second approach is a Mori-Tanaka-based approach in which the interaction tensors are calculated using the modified Eshelby's heterogeneity problem. The interaction tensors link the strain in the particle with the strain in the matrix.

Remark. The case of long fiber composites with interface effects has been studied by the authors (Firooz et al., 2019a). For short fiber composites, however, analytical solutions to boundary value problems similar to the ones examined here are not available. Thus one should use other methods for studying them such as mean field, e.g. MoriTanaka. It is important to note that, the presence of the imperfect interface renders a nonuniform Eshelby tensor for interfaces with nonconstant curvature (Sharma and Ganti, 2004) and therefore, certain simplifications and assumptions are unavoidable for short fiber composites. Dinzart and Sabar (2017) have applied a similar strategy for composites considering only the displacement jump at the interface by assuming that the stress field at the inclusion remains uniform, which is not generally true and accurate for imperfect interfaces.

\subsection{Preliminaries of the RVE problem for particulate composites}

Fig. 4 shows a heterogeneous medium with its underlying microstructure as well as a proper coordinate system to examine such medium. The simplified RVE consists of two concentric spheres corresponding to the matrix and the particle with the general interface lying at $r=r_{1}$. In passing, it proves convenient to express the homogenization problem in this medium in spherical coordinate system with the coordinates $r, \theta, \phi$. Throughout this manuscript, the quantities 

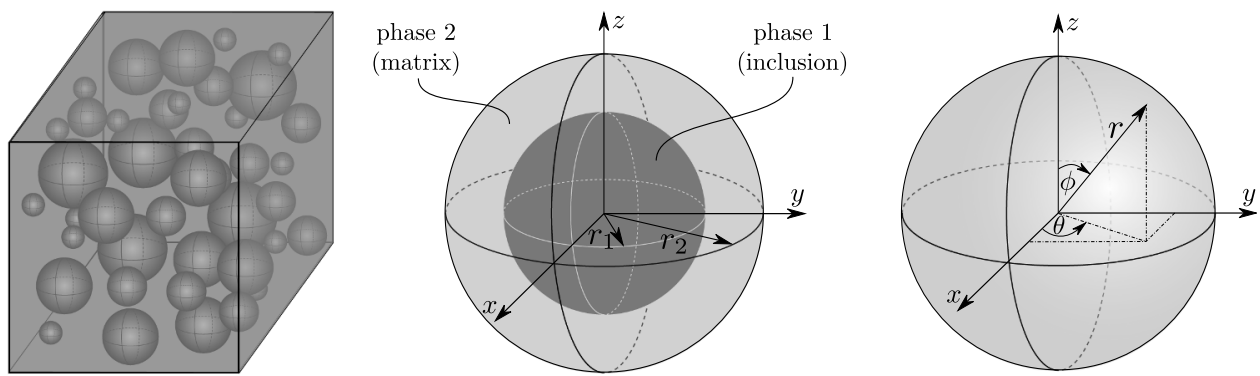

Fig. 4. Heterogeneous medium (left) with its simplified RVE (middle) and the proper coordinate system (right) to examine such medium.

corresponding to the particle are designated by index 1 while index 2 indicates the matrix-related quantities. The volume fraction of the particle is $f=r_{1}^{3} / r_{2}^{3}$.

The constitutive material behavior in Voigt notation reads

$$
\begin{aligned}
{\left[\begin{array}{c}
\sigma_{r r} \\
\sigma_{\theta \theta} \\
\sigma_{\phi \phi} \\
\sigma_{r \theta} \\
\sigma_{r \phi} \\
\sigma_{\theta \phi}
\end{array}\right] } & =\left[\begin{array}{cccccc}
\kappa+4 \mu / 3 & \kappa-2 \mu / 3 & \kappa-2 \mu / 3 & 0 & 0 & 0 \\
\kappa-2 \mu / 3 & \kappa+4 \mu / 3 & \kappa-2 \mu / 3 & 0 & 0 & 0 \\
\kappa-2 \mu / 3 & \kappa-2 \mu / 3 & \kappa+4 \mu / 3 & 0 & 0 & 0 \\
0 & 0 & 0 & \mu & 0 & 0 \\
0 & 0 & 0 & 0 & \mu & 0 \\
0 & 0 & 0 & 0 & 0 & \mu
\end{array}\right]\left[\begin{array}{c}
\varepsilon_{r r} \\
\varepsilon_{\theta \theta} \\
\varepsilon_{\phi \phi} \\
2 \varepsilon_{r \theta} \\
2 \varepsilon_{r \phi} \\
2 \varepsilon_{\theta \phi}
\end{array}\right], \\
\varepsilon_{r r} & =\frac{\partial u_{r}}{\partial r}, \\
\varepsilon_{\theta \theta} & =\frac{1}{r} \frac{\partial u_{\theta}}{\partial \theta}+\frac{u_{r}}{r}, \\
\varepsilon_{\phi \phi} & =\frac{1}{r \sin \theta} \frac{\partial u_{\phi}}{\partial \phi}+\frac{u_{r}}{r}+\frac{u_{\theta} \cos \theta}{r \sin \theta}, \\
2 \varepsilon_{r \phi} & =\frac{\partial u_{\phi}}{\partial r}+\frac{1}{r \sin \theta} \frac{\partial u_{r}}{\partial \phi}-\frac{u_{\phi}}{r}, \\
2 \varepsilon_{\theta \phi} & =\frac{1}{r} \frac{\partial u_{\phi}}{\partial \theta}+\frac{1}{r \sin \theta} \frac{\partial u_{\theta}}{\partial \phi}-\frac{u_{\phi} \cos \theta}{r \sin \theta}, \\
2 \varepsilon_{r \theta} & =\frac{\partial u_{\theta}}{\partial r}+\frac{1}{r} \frac{\partial u_{r}}{\partial \theta}-\frac{u_{\theta}}{r},
\end{aligned}
$$

and the equilibrium equations in the bulk are

$$
\left\{\begin{array}{l}
\frac{\partial \sigma_{r r}}{\partial r}+\frac{1}{r} \frac{\partial \sigma_{r \theta}}{\partial \theta}+\frac{\sigma_{r \theta} \cos \theta}{r \sin \theta}+\frac{2 \sigma_{r r}-\sigma_{\theta \theta}-\sigma_{\phi \phi}}{r}+\frac{1}{r \sin \theta} \frac{\partial \sigma_{r \phi}}{\partial \phi}=0, \\
\frac{\partial \sigma_{r \theta}}{\partial r}+\frac{1}{r} \frac{\partial \sigma_{\theta \theta}}{\partial \theta}+\frac{3 \sigma_{r \theta}}{r}+\frac{\left[\sigma_{\theta \theta}-\sigma_{\phi \phi}\right] \cos \theta}{r \sin \theta}+\frac{1}{r \sin \theta} \frac{\partial \sigma_{\theta \phi}}{\partial \phi}=0, \\
\frac{\partial \sigma_{r \phi}}{\partial r}+\frac{1}{r} \frac{\partial \sigma_{\theta \phi}}{\partial \theta}+\frac{3 \sigma_{r \phi}}{r}+\frac{2 \sigma_{\theta \phi} \cos \theta}{r \sin \theta}+\frac{1}{r \sin \theta} \frac{\partial \sigma_{\phi \phi}}{\partial \phi}=0 .
\end{array}\right.
$$

Considering the spherical particles having the radius $r=r_{1}$, the constitutive relations at the interface read

with the interface equilibrium equations

$$
\left\{\begin{array}{l}
-\frac{\bar{\sigma}_{\theta \theta}+\bar{\sigma}_{\phi \phi}}{r_{1}}+\left[\left[\sigma_{r r}\right]\right]=0, \\
\frac{1}{r_{1}} \frac{\partial \bar{\sigma}_{\theta \theta}}{\partial \theta}+\frac{1}{r_{1} \sin \theta} \frac{\partial \bar{\sigma}_{\theta \phi}}{\partial \phi}+\frac{\left[\bar{\sigma}_{\theta \theta}-\bar{\sigma}_{\phi \phi}\right] \cos \theta}{r_{1} \sin \theta}+\left[\left[\sigma_{r \theta}\right]\right]=0, \\
\frac{1}{r_{1}} \frac{\partial \bar{\sigma}_{\theta \phi}}{\partial \theta}+\frac{1}{r_{1} \sin \theta} \frac{\partial \bar{\sigma}_{\phi \phi}}{\partial \phi}+\frac{2 \bar{\sigma}_{\theta \phi} \cos \theta}{r_{1} \sin \theta}+\left[\left[\sigma_{r \phi}\right]\right]=0 .
\end{array}\right.
$$

The displacements and stresses can be presented more precisely as

$$
\begin{aligned}
& \boldsymbol{u}=u_{r} \boldsymbol{n}_{r}+u_{\theta} \boldsymbol{n}_{\theta}+u_{\phi} \boldsymbol{n}_{\phi}, \\
& \sigma_{r r} \boldsymbol{n}_{r} \otimes \boldsymbol{n}_{r}+\sigma_{\theta \theta} \boldsymbol{n}_{\theta} \otimes \boldsymbol{n}_{\theta}+\sigma_{\phi \phi} \boldsymbol{n}_{\phi} \otimes \boldsymbol{n}_{\phi}+\frac{1}{2} \sigma_{r \theta} \\
& \sigma=\quad\left[n_{r} \otimes n_{\theta}+n_{\theta} \otimes n_{r}\right] \\
& +\frac{1}{2} \sigma_{r \phi}\left[\boldsymbol{n}_{r} \otimes \boldsymbol{n}_{\phi}+\boldsymbol{n}_{\phi} \otimes \boldsymbol{n}_{r}\right]+\frac{1}{2} \sigma_{\theta \phi} \\
& {\left[n_{\theta} \otimes n_{\phi}+n_{\phi} \otimes n_{\theta}\right] \text {, }} \\
& \bar{\sigma}=\bar{\sigma}_{\theta \theta} \boldsymbol{n}_{\theta} \otimes \boldsymbol{n}_{\theta}+\bar{\sigma}_{\phi \phi} \boldsymbol{n}_{\phi} \otimes \boldsymbol{n}_{\phi}+\frac{1}{2} \bar{\sigma}_{\theta \phi}\left[\boldsymbol{n}_{\theta} \otimes \boldsymbol{n}_{z}+\boldsymbol{n}_{z} \otimes \boldsymbol{n}_{\theta}\right],
\end{aligned}
$$

with the normal vectors in spherical coordinates

$\boldsymbol{n}_{r}=\left[\begin{array}{c}\sin \theta \cos \phi \\ \sin \theta \sin \phi \\ \cos \theta\end{array}\right], \quad \boldsymbol{n}_{\theta}=\left[\begin{array}{c}\cos \theta \cos \phi \\ \cos \theta \sin \phi \\ -\sin \theta\end{array}\right], \quad \boldsymbol{n}_{\phi}=\left[\begin{array}{c}-\sin \phi \\ \cos \phi \\ 0\end{array}\right]$.

Finally, the overall mechanical energy stored in the RVE and in equivalent homogeneous medium read

$$
\begin{aligned}
U^{\mathrm{RVE}} & =\frac{1}{2 \mathscr{V}} \int_{\mathcal{B}} \sigma \cdot \varepsilon \mathrm{d} V \\
& =\frac{3}{8 \pi r_{2}} \int_{0}^{2 \pi} \int_{0}^{\pi}\left[\sigma_{r r}^{(2)} u_{r}^{(2)}+\sigma_{r \theta}^{(2)} u_{\theta}^{(2)}+\sigma_{r \phi}^{(2)} u_{\phi}^{(2)}\right]_{r=r_{2}} \sin \theta \mathrm{d} \theta \mathrm{d} \phi, \\
U^{\mathrm{eq}} & =\frac{1}{2 \mathscr{V}} \int_{\mathcal{B}} \sigma \cdot \varepsilon \mathrm{d} V \\
& =\frac{3}{8 \pi r_{2}} \int_{0}^{2 \pi} \int_{0}^{\pi}\left[\sigma_{r r}^{\mathrm{eq}} u_{r}^{\mathrm{eq}}+\sigma_{r \theta}^{\mathrm{eq}} u_{\theta}^{\mathrm{eq}}+\sigma_{r \phi}^{\mathrm{eq}} u_{\phi}^{\mathrm{eq}}\right]_{r=r_{2}} \sin \theta \mathrm{d} \theta \mathrm{d} \phi .
\end{aligned}
$$

$$
\begin{aligned}
& \bar{\varepsilon}_{\theta \theta}=\frac{1}{r_{1}} \frac{\partial \bar{u}_{\theta}}{\partial \theta}+\frac{\bar{u}_{r}}{r_{1}}, \\
& \bar{\varepsilon}_{\phi \phi}=\frac{1}{r_{1} \sin \theta} \frac{\partial \bar{u}_{\phi}}{\partial \phi}+\frac{\bar{u}_{r}}{r_{1}}+\frac{\bar{u}_{\theta} \cos \theta}{r_{1} \sin \theta}, \\
& 2 \bar{\varepsilon}_{\theta \phi}=\frac{1}{r_{1}} \frac{\partial \bar{u}_{\phi}}{\partial \theta}+\frac{1}{r_{1} \sin \theta} \frac{\partial \bar{u}_{\theta}}{\partial \phi}-\frac{\bar{u}_{\phi} \cos \theta}{r_{1} \sin \theta},
\end{aligned}
$$$$
\left[\begin{array}{c}
\bar{\sigma}_{\theta \theta} \\
\bar{\sigma}_{\phi \phi} \\
\bar{\sigma}_{\theta \phi}
\end{array}\right]=\left[\begin{array}{ccc}
\bar{\lambda}+2 \bar{\mu} & \bar{\lambda} & 0 \\
\bar{\lambda} & \bar{\lambda}+2 \bar{\mu} & 0 \\
0 & 0 & \bar{\mu}
\end{array}\right]\left[\begin{array}{c}
\bar{\varepsilon}_{\theta \theta} \\
\bar{\varepsilon}_{\phi \phi} \\
2 \bar{\varepsilon}_{\theta \phi}
\end{array}\right], \quad \bar{\varepsilon}_{\phi \phi}=\frac{1}{r_{1} \sin \theta} \frac{\partial \bar{u}_{\phi}}{\partial \phi}+\frac{\bar{u}_{r}}{r_{1}}+\frac{\bar{u}_{\theta} \cos \theta}{r_{1} \sin \theta}, \quad\left[\begin{array}{c}
\bar{t}_{r} \\
\bar{t}_{\theta} \\
\bar{t}_{\phi}
\end{array}\right]=\bar{k}\left[\begin{array}{l}
\llbracket u_{r} \rrbracket \\
\llbracket u_{\theta} \rrbracket \\
\llbracket u_{\phi} \rrbracket
\end{array}\right],
$$ 


\subsection{Composite sphere assemblage (CSA) approach and the generalized self-} consistent method (GSCM)

The original CSA approach was developed by Hashin (1962) where he provided analytical solution strategies to estimate the macroscopic bulk modulus ${ }^{{ }}{ }_{k}$ and the macroscopic shear modulus ${ }^{{ }} \mu$ of a particulate composite. While the original CSA approach can accurately calculate the effective bulk modulus, it can only provide bounds on the effective shear modulus. Christensen and Lo (1979) resolved this issue via solving the boundary value problem using the generalized self consistent method and obtained an estimation of the effective shear modulus. In this case, the RVE is assumed as a set of three concentric spheres where the external layer is an unknown effective medium. We extend these two approaches to account for the general interfaces and derive explicit expressions for the overall bulk modulus $\mathrm{M}_{\boldsymbol{k}}$ and semi-explicit expressions for the upper bound, lower bound and an estimate for the overall shear modulus $\mathrm{M}_{\mu}$ of particle reinforced composites.

\subsubsection{Effective bulk modulus}

Assume that the RVE is subject to a far field hydrostatic displacement field. The displacement in both Cartesian and spherical coordinates read

$\boldsymbol{u}_{(x, y, z)}^{0}=\left[\begin{array}{c}\beta x \\ \beta y \\ \beta z\end{array}\right] \quad$ and $\quad \boldsymbol{u}_{(r, \theta, \phi)}^{0}=\left[\begin{array}{c}\beta r \\ 0 \\ 0\end{array}\right]$.

For this type of boundary condition, Hashin (1962) has demonstrated that at every phase the displacement field that satisfies the equilibrium Eq. (11) reads

$$
\begin{aligned}
u_{r}^{(i)} & =\beta r U_{r}^{(i)}(r), \quad u_{\theta}^{(i)}=u_{\phi}^{(i)}=0 \quad \text { with } U_{r}^{(i)}(r) \\
& =\Xi_{1}^{(i)}+\Xi_{2}^{(i)} \frac{1}{\left[r / r_{1}\right]^{3}},
\end{aligned}
$$

for $i=1,2$ where $i=1$ corresponds to the particle and $i=2$ corresponds to the matrix. The unknowns $\Xi_{1}^{(2)}, \Xi_{2}^{(2)}, \Xi_{1}^{(1)}$ and $\Xi_{2}^{(1)}$ can be calculated using the boundary and interface conditions

$$
\begin{aligned}
& u_{r}^{(1)} \text { finite at } r=0 \rightarrow \Xi_{2}^{(1)}=0, \\
& \text { (finite displacement at } r \\
& =0) \\
& \bar{t}_{r}=\bar{k}_{r}\left[\left[u_{r}\right]\right] \quad \rightarrow \frac{\sigma_{r r}^{(2)}\left(r_{1}\right)+\sigma_{r r}^{(1)}\left(r_{1}\right)}{2} \text { (traction average at } r=r_{1} \\
& =\bar{k}_{r}\left[u_{r}^{(2)}\left(r_{1}\right)\right. \\
& \left.-u_{r}^{(1)}\left(r_{1}\right)\right] \text {, } \\
& {[\overline{\operatorname{div}} \bar{\sigma}]_{r}+\left[\left[t_{r}\right]\right]=0 \rightarrow-\frac{\bar{\sigma}_{\theta \theta}+\bar{\sigma}_{\phi \phi}}{r_{1}} \quad \text { (traction equilibrium at } r} \\
& +\sigma_{r r}^{(2)}\left(r_{1}\right) \\
& \left.=r_{1}\right) \\
& \text { - } \sigma_{r r}^{(1)}\left(r_{1}\right)=0 \text {, }
\end{aligned}
$$

leading to the system

$\left[\begin{array}{ccc}1+\frac{3 \kappa_{1}}{2 \bar{k} r_{1}} & -1+\frac{3 \kappa_{2}}{2 \bar{k} r_{1}} & -1-\frac{2 \mu_{2}}{\bar{k} r_{1}} \\ 3 \kappa_{1}+\frac{2[\bar{\lambda}+\bar{\mu}]}{r_{1}} & -3 \kappa_{2}+\frac{2[\bar{\lambda}+\bar{\mu}]}{r_{1}} & 4 \mu_{2}+\frac{2[\bar{\lambda}+\bar{\mu}]}{r_{1}} \\ 0 & 1 & f\end{array}\right]\left[\begin{array}{l}\Xi_{1}^{(1)} \\ \Xi_{1}^{(2)} \\ \Xi_{2}^{(2)}\end{array}\right]=\left[\begin{array}{l}0 \\ 0 \\ 1\end{array}\right]$.

If the RVE is substituted by an equivalent homogeneous medium, applying the boundary condition (17) yields the displacement field $u_{r}^{\mathrm{eq}}=\beta r$ and $u_{\theta}^{\mathrm{eq}}=u_{\phi}^{\mathrm{eq}}=0$. We assume isotropic response for the interface and thus, $\bar{k}_{r}=\bar{k}_{\theta}=\bar{k}_{\phi}=\bar{k}$. Using Eq. (16), the overall energy in both the RVE and the equivalent homogeneous medium read

$U^{\mathrm{RVE}}=\frac{3 \beta^{2}}{2}\left[3 \kappa_{2} \Xi_{1}^{(2)}-4 f \mu_{2} \Xi_{2}^{(2)}\right] \quad$ and $\quad U^{\mathrm{eq}}=\frac{9 \beta^{2}}{2} \mathrm{M}_{\mathcal{K}}$.

According to the Hill-Mandel condition, the above energies should be equal. Therefore, we obtain a closed-form expression for the macroscopic bulk modulus ${ }^{M_{k}}$ of particulate composites with general interfaces

$$
\begin{aligned}
& {\left[\bar{\lambda}+\bar{\mu}+\bar{k} r_{1}^{2}\right]\left[3 \kappa_{1}\left[3 \kappa_{2}+4 f \mu_{2}\right]+12 \kappa_{2} \mu_{2}[1-f]\right]+4 \bar{k} r_{1}} \\
& \mathrm{M}_{k}=\frac{[\bar{\lambda}+\bar{\mu}]\left[3 \kappa_{2}+4 f \mu_{2}\right]+36 \kappa_{1} \kappa_{2} \mu_{2} r_{1}[1-f]}{3\left[\bar{\lambda}+\bar{\mu}+\bar{k} r_{1}^{2}\right]\left[3 \kappa_{1}+4 \mu_{2}+3 f\left[\kappa_{2}-\kappa_{1}\right]\right]+12 \bar{k} r_{1}} \\
& {[\bar{\lambda}+\bar{\mu}][1-f]+9 \kappa_{1} r_{1}\left[4 \mu_{2}+3 f \kappa_{2}\right]}
\end{aligned}
$$

\subsubsection{Strain bound on shear modulus}

For this case, consider an RVE subject to a deviatoric displacement field. The displacement in both Cartesian and spherical coordinates read

$\boldsymbol{u}_{(x, y, z)}^{0}=\left[\begin{array}{c}\beta y \\ -\beta x \\ 0\end{array}\right]$ and $\quad \boldsymbol{u}_{(r, \theta, \phi)}^{0}=\left[\begin{array}{c}\beta r \sin ^{2} \theta \cos 2 \phi \\ \beta r \sin \theta \cos \theta \cos 2 \phi \\ -\beta r \sin \theta \sin 2 \phi\end{array}\right]$.

For this type of boundary condition, Christensen and Lo (1979) have demonstrated that at every phase the displacement field that satisfies the equilibrium Eq. (11) reads

$$
\begin{aligned}
& u_{r}^{(i)}=\beta r U_{r}^{(i)}(r) \sin ^{2} \theta \cos 2 \phi, \\
& u_{\theta}^{(i)}=\beta r U_{\theta}^{(i)}(r) \sin \theta \cos \theta \cos 2 \phi, \\
& u_{\phi}^{(i)}=-\beta r U_{\theta}^{(i)}(r) \sin \theta \sin 2 \phi, \\
& \text { with }\left\{\begin{array}{l}
U_{r}^{(i)}(r)=\Xi_{1}^{(i)}+\left[2-3 \frac{\kappa_{i}}{\mu_{i}}\right]\left[r / r_{1}\right]^{2} \Xi_{2}^{(i)}+\frac{3 \Xi_{3}^{(i)}}{\left[r / r_{1}\right]^{5}}+ \\
\quad\left[3+3 \frac{\kappa_{i}}{\mu_{i}}\right] \frac{\Xi_{4}^{(i)}}{\left[r / r_{1}\right]^{3}}, \\
U_{\theta}^{(i)}(r)=\Xi_{1}^{(i)}-\left[\frac{11}{3}+5 \frac{\kappa_{i}}{\mu_{i}}\right]\left[r / r_{1}\right]^{2} \Xi_{2}^{(i)}-\frac{2 \Xi_{3}^{(i)}}{\left[r / r_{1}\right]^{5}}+\frac{2 \Xi_{4}^{(i)}}{\left[r / r_{1}\right]^{3}} .
\end{array}\right.
\end{aligned}
$$


The eight unknowns $\Xi_{1}^{(1)}, \Xi_{2}^{(1)}, \Xi_{3}^{(1)}, \Xi_{4}^{(1)}, \Xi_{1}^{(2)}, \Xi_{2}^{(2)}, \Xi_{3}^{(2)}, \Xi_{4}^{(2)}$ can be calculated using the boundary and interface conditions
Further details regarding the construction of the system of equations are available in Appendix A.2. If the RVE is substituted by an equivalent

$\begin{array}{lll}u_{r}^{(1)}, u_{\theta}^{(1)} \text { finite at } r=0 & \rightarrow \Xi_{3}^{(1)}=\Xi_{4}^{(1)}=0, & \text { finite displacement at } r=0 \\ \begin{array}{l}\bar{t}_{r}=\bar{k}_{r}\left[\left[u_{r}\right]\right] \\ \bar{t}_{\theta}=\bar{k}_{\theta}\left[\left[u_{\theta}\right]\right]\end{array} & \rightarrow \sigma_{r r}^{(2)}\left(r_{1}\right)+\sigma_{r r}^{(1)}\left(r_{1}\right)=2 \bar{k}_{r}\left[u_{r}^{(2)}\left(r_{1}\right)-u_{r}^{(1)}\left(r_{1}\right)\right], & \left.\text { (traction average at } r=r_{1}\right) \\ {[\overline{\operatorname{div}} \bar{\sigma}]_{r}+\left[\left[t_{r}\right]\right]=0} & \rightarrow-\frac{\sigma_{r \theta}^{(2)}\left(r_{1}\right)+\sigma_{r \theta}^{(1)}\left(r_{1}\right)=2 \bar{k}_{\theta}\left[u_{\theta}^{(2)}\left(r_{1}\right)-u_{\theta}^{(1)}\left(r_{1}\right)\right],}{\left.\text { (traction average at } r=r_{1}\right)} \\ & \rightarrow \frac{1}{r_{1}} \frac{\partial \bar{\sigma}_{\theta \theta}}{\partial \theta}+\frac{1}{r_{1} \sin \theta} \frac{\partial \bar{\sigma}_{\theta \phi}}{\partial \phi}+\frac{\left[\bar{\sigma}_{\theta \theta}-\bar{\sigma}_{\phi \phi}\right] \cos \theta}{r_{1} \sin \theta} & \left.\text { (traction equilibrium at } r=r_{1}\right) \\ {[\overline{\operatorname{div}} \bar{\sigma}]_{\theta}+\left[\left[t_{\theta}\right]\right]=0} & \quad+\sigma_{r \theta}^{(2)}\left(r_{1}\right)-\sigma_{r \theta}^{(1)}\left(r_{1}\right)=0, & \left.\text { (traction equilibrium at } r=r_{1}\right) \\ u_{r}^{(2)}\left(r=r_{2}\right)=\beta r \sin ^{2} \theta \cos 2 \phi, & u_{\theta}^{(2)}\left(r=r_{2}\right)=\beta r \sin \theta \cos \theta \cos 2 \phi, & \left.\text { (displacement at } r=r_{2}\right)\end{array}$

Further details regarding the construction of the system of equations are available in Appendix A.1. If the RVE is substituted by an equivalent homogeneous medium, applying the same boundary condition leads to the displacement field

$u_{r}^{\mathrm{eq}}=\beta r \sin ^{2} \theta \cos 2 \phi, \quad u_{\theta}^{\mathrm{eq}}=\beta r \sin \theta \cos \theta \cos 2 \phi$,

$u_{\phi}^{\mathrm{eq}}=-\beta r \sin \theta \sin 2 \phi$.

Equipped with all the displacement and stress fields, the overall energy according to Eq. (16) in both RVE and the equivalent homogeneous medium read

$$
\begin{aligned}
U^{\mathrm{RVE}}= & \frac{\beta^{2}}{5}\left[10 \mu_{2} \Xi_{1}^{(2)}-14\left[3 \kappa_{2}+\mu_{2}\right] f^{-2 / 3} \Xi_{2}^{(2)}-2\left[9 \kappa_{2}+8 \mu_{2}\right] f \Xi_{4}^{(2)}\right] \\
& \text { and } \quad U^{\mathrm{eq}}=2 \beta^{2 \mathrm{M}} \mu .
\end{aligned}
$$

Considering $U^{\mathrm{RVE}}=U^{\mathrm{eq}}$ results in a semi-explicit expression for the strain bound on the macroscopic shear modulus

$\mathrm{M}_{\mu_{\text {strain }}}=\frac{1}{10}\left[10 \mu_{2} \Xi_{1}^{(2)}-14\left[3 \kappa_{2}+\mu_{2}\right] f^{-2 / 3} \Xi_{2}^{(2)}-2\left[9 \kappa_{2}+8 \mu_{2}\right] f \Xi_{4}^{(2)}\right]$,

where $\Xi_{1}^{(2)}, \Xi_{2}^{(2)}$ and $\Xi_{4}^{(2)}$ are the solutions of the system of Eq. (A.1).

\subsubsection{Stress bound on shear modulus}

In order to obtain the stress bound, unlike the strain bound due to prescribed displacements, we prescribe traction on the RVE. Hence, consider an RVE subject to the traction field

$\boldsymbol{t}_{(r, \theta, \phi)}^{0}=\left[\begin{array}{c}\sigma_{r r}^{0} \\ \sigma_{r \theta}^{0} \\ \sigma_{r \phi}^{0}\end{array}\right]=\left[\begin{array}{c}\beta \sin ^{2} \theta \cos 2 \phi \\ \beta \sin \theta \cos \theta \cos 2 \phi \\ -\beta \sin \theta \sin 2 \phi\end{array}\right]$.

The solution of the boundary value problem for this case is similar to Eq. (24) and the eight unknowns $\Xi_{1}^{(1)}, \Xi_{2}^{(1)}, \Xi_{3}^{(1)}, \Xi_{4}^{(1)}, \Xi_{1}^{(2)}, \Xi_{2}^{(2)}, \Xi_{3}^{(2)}$ and $\Xi_{4}^{(2)}$ can be calculated using the boundary and interface conditions homogeneous medium, applying the boundary condition (29) results in the displacement field

$u_{r}^{\mathrm{eq}}=\frac{\beta}{2^{\mathrm{M}_{\mu}}} r \sin ^{2} \theta \cos 2 \phi, \quad u_{\theta}^{\mathrm{eq}}=\frac{\beta}{2^{\mathrm{M}_{\mu}}} r \sin \theta \cos \theta \cos 2 \phi$,

$u_{\phi}^{\mathrm{eq}}=-\frac{\beta}{2^{\mathrm{M}} \mu} r \sin \theta \sin 2 \phi$.

Having all the displacement and stress fields, according to Eq. (16), the overall energy in both RVE and the equivalent homogeneous medium read

$$
\begin{gathered}
U^{\mathrm{RVE}}=\frac{\beta^{2}}{5}\left[5 \Xi_{1}^{(2)}-7\left[1+3 \frac{\kappa_{2}}{\mu_{2}}\right] f^{-2 / 3} \Xi_{2}^{(2)}+6\left[2+\frac{\kappa_{2}}{\mu_{2}}\right] f \Xi_{4}^{(2)}\right] \\
U^{\mathrm{eq}}=\frac{\beta^{2}}{2^{\mathrm{M} \mu}},
\end{gathered}
$$

resulting in a semi-explicit expression for the stress bound on the effective shear modulus

$\mathrm{M}_{\mu_{\text {stress }}}=\frac{5}{2}\left[5 \Xi_{1}^{(2)}-7\left[1+3 \frac{\kappa_{2}}{\mu_{2}}\right] f^{-2 / 3} \Xi_{2}^{(2)}+6\left[2+\frac{\kappa_{2}}{\mu_{2}}\right] f \Xi_{4}^{(2)}\right]^{-1}$,

where $\Xi_{1}^{(2)}, \Xi_{2}^{(2)}$ and $\Xi_{4}^{(2)}$ are the solutions of the system of Eq. (A.3).

\subsubsection{Effective shear modulus}

In order to obtain the effective shear modulus $\mathrm{M}_{\mu}$ of a particle reinforced composite, we employ the method proposed by Christensen and Lo (1979) considering an infinite effective medium surrounding the matrix whose properties are the unknowns of our problem. Consider the RVE subject to a deviatoric displacement field

$\boldsymbol{u}_{(x, y, z)}^{0}=\left[\begin{array}{c}\beta y \\ -\beta x \\ 0\end{array}\right]$ and $u_{(r, \theta, \phi)}^{0}=\left[\begin{array}{c}\beta r \sin ^{2} \theta \cos 2 \phi \\ \beta r \sin \theta \cos \theta \cos 2 \phi \\ -\beta r \sin \theta \sin 2 \phi\end{array}\right]$.

(finite displacement at $r=0$ )

(traction average at $r=r_{1}$ )

(traction average at $r=r_{1}$ )

(traction equilibrium at $r=r_{1}$ )

(traction equilibrium at $r=r_{1}$ )

(traction at $r=r_{2}$ ) 
The displacement field in the particle and the matrix for this type of boundary condition is similar to Eq. (24), see Christensen and Lo (1979) for further details. The displacement field in the effective medium reads

$u_{r}^{\text {(eff) }}(r, \theta, \phi)=\beta r U_{r}^{\text {(eff) }}(r) \sin ^{2} \theta \cos 2 \phi$,

$u_{\theta}^{\text {(eff) }}(r, \theta, \phi)=\beta r U_{\theta}^{\text {(eff) }}(r) \sin \theta \cos \theta \cos 2 \phi$,

$u_{\phi}^{\text {(eff) }}(r, \theta, \phi)=-\beta r U_{\theta}^{\text {(eff) }}(r) \sin \theta \sin 2 \phi$,

with $\left\{\begin{array}{l}U_{r}^{\text {(eff) }}(r)=1+\frac{3 \Xi_{3}^{(\text {eff })}}{\left[r / r_{1}\right]^{5}}+\left[3+3 \frac{\mathrm{M}_{k}}{\mathrm{M}_{\mu}}\right] \frac{\Xi_{4}^{(\text {eff })}}{\left[r / r_{1}\right]^{3}}, \\ U_{\theta}^{\text {(eff) }}(r)=1-\frac{2 \Xi_{3}^{(\text {eff })}}{\left[r / r_{1}\right]^{5}}+\frac{2 \Xi_{4}^{(\text {eff }}}{\left[r / r_{1}\right]^{3}} .\end{array}\right.$

Perfect bonding between the effective medium and the matrix is assumed which renders

$$
\begin{array}{lll}
u_{r}^{(2)}\left(r_{2}, \theta, \phi\right) & u_{\theta}^{(2)}\left(r_{2}, \theta, \phi\right) & u_{\phi}^{(2)}\left(r_{2}, \theta, \phi\right) \\
=u_{r}^{(\text {eff }}\left(r_{2}, \theta, \phi\right), & =u_{\theta}^{(\mathrm{eff})}\left(r_{2}, \theta, \phi\right), & =u_{\phi}^{(\mathrm{eff})}\left(r_{2}, \theta, \phi\right), \\
\sigma_{r r}^{(2)}\left(r_{2}, \theta, \phi\right) & \sigma_{r \theta}^{(2)}\left(r_{2}, \theta, \phi\right) & \sigma_{r \phi}^{(2)}\left(r_{2}, \theta, \phi\right) \\
=\sigma_{r r}^{(\mathrm{eff})}\left(r_{2}, \theta, \phi\right), & =\sigma_{r \theta}^{(\mathrm{eff})}\left(r_{2}, \theta, \phi\right), & =\sigma_{r \phi}^{(\mathrm{eff})}\left(r_{2}, \theta, \phi\right) .
\end{array}
$$

So far our problem contains ten unknowns of $\Xi_{1}^{(1)}, \Xi_{2}^{(1)}, \Xi_{3}^{(1)}, \Xi_{4}^{(1)}, \Xi_{1}^{(2)}$, $\Xi_{2}^{(2)}, \Xi_{3}^{(2)}, \Xi_{4}^{(2)}, \Xi_{3}^{(\text {eff })}$ and $\Xi_{4}^{\text {(eff) }}$ which can be calculated using the boundary and interface conditions

$$
\begin{aligned}
& u_{r}^{(1)}, u_{\theta}^{(1)} \text { finite at } r=0 \rightarrow \Xi_{3}^{(1)}=\Xi_{4}^{(1)}=0 \text {, } \\
& \bar{t}_{r}=\bar{k}_{r}\left[\left[u_{r}\right]\right] \\
& \rightarrow \sigma_{r r}^{(2)}\left(r_{1}\right)+\sigma_{r r}^{(1)}\left(r_{1}\right)=2 \bar{k}_{r}\left[u_{r}^{(2)}\left(r_{1}\right)-u_{r}^{(1)}\left(r_{1}\right)\right] \text {, } \\
& \bar{t}_{\theta}=\bar{k}_{\theta}\left[\left[u_{\theta}\right]\right] \\
& \rightarrow \sigma_{r \theta}^{(2)}\left(r_{1}\right)+\sigma_{r \theta}^{(1)}\left(r_{1}\right)=2 \bar{k}_{\theta}\left[u_{\theta}^{(2)}\left(r_{1}\right)-u_{\theta}^{(1)}\left(r_{1}\right)\right] \text {, } \\
& {[\overline{\operatorname{div}} \bar{\sigma}]_{r}+\left[\left[t_{r}\right]\right]=0} \\
& \rightarrow-\frac{\bar{\sigma}_{\theta \theta}+\bar{\sigma}_{\phi \phi}}{r_{1}}+\sigma_{r r}^{(2)}\left(r_{1}\right)-\sigma_{r r}^{(1)}\left(r_{1}\right)=0, \\
& {[\overline{\operatorname{div}} \bar{\sigma}]_{\theta}+\left[\left[t_{\theta}\right]\right]=0} \\
& \rightarrow \frac{1}{r_{1}} \frac{\partial \bar{\sigma}_{\theta \theta}}{\partial \theta}+\frac{1}{r_{1} \sin \theta} \frac{\partial \bar{\sigma}_{\theta \phi}}{\partial \phi}+\frac{\left[\bar{\sigma}_{\theta \theta}-\bar{\sigma}_{\phi \phi}\right] \cos \theta}{r_{1} \sin \theta} \\
& +\sigma_{r \theta}^{(2)}\left(r_{1}\right)-\sigma_{r \theta}^{(1)}\left(r_{1}\right)=0, \\
& \sigma_{r r}^{(2)}\left(r_{2}\right)=\sigma_{r r}^{(\text {eff })}\left(r_{2}\right), \\
& \sigma_{r \theta}^{(2)}\left(r_{2}\right)=\sigma_{r \theta}^{(\mathrm{eff})}\left(r_{2}\right), \\
& u_{r}^{(2)}\left(r_{2}\right)=u_{r}^{(\mathrm{eff})}\left(r_{2}\right) \text {, } \\
& u_{\theta}^{(2)}\left(r_{2}\right)=u_{\theta}^{(\mathrm{eff})}\left(r_{2}\right) \text {. }
\end{aligned}
$$

Further details regarding the construction of the system of equations are available in Appendix A.3. Considering a homogeneous equivalent medium under the same boundary condition, the displacement field in the medium is similar to Eq. (26). From the Eshelby's energy principle, we can deduce

$\int_{0}^{2 \pi} \int_{0}^{\pi}\left[\sigma_{r r}^{(\text {eff })} u_{r}^{\mathrm{eq}}+\sigma_{r \theta}^{(\mathrm{eff})} u_{\theta}^{\mathrm{eq}}+\sigma_{r \phi}^{(\mathrm{eff})} u_{\phi}^{\mathrm{eq}}-\sigma_{r r}^{\mathrm{eq}} u_{r}^{(\mathrm{eff})}-\sigma_{r \theta}^{\mathrm{eq}} u_{\theta}^{(\mathrm{eff})}-\sigma_{r \phi}^{\mathrm{eq}} u_{\phi}^{(\mathrm{eff})}\right]_{r=r_{2}}$ $\sin \theta \mathrm{d} \theta \mathrm{d} \phi=0$

Substituting the stress and displacement fields of the RVE and equivalent homogeneous medium at $r=r_{2}$ in this integral results in $\Xi_{4}^{\text {(eff) }}=0$. Unlike the macroscopic bulk modulus ${ }^{k_{k}}$, it is not possible to provide an explicit expression for the effective shear modulus $\mathrm{M}_{\mu}$ and identify its simplified forms for various interface types. Nonetheless, we have developed the following semi-explicit expression to obtain the macroscopic shear modulus $\mathrm{M}_{\mu}$

$$
\begin{aligned}
\mathrm{M}_{\mu} & =\frac{1}{80}\left[b_{6}-b_{5}+12 a_{6}+8 a_{5}+\sqrt{\Delta}\right] \text { with } \Delta \\
& =\left[b_{6}-b_{5}+12 a_{6}+8 a_{5}\right]^{2}-80\left[a_{5} b_{6}-b_{5} a_{6}\right],
\end{aligned}
$$

where the constants $a_{5}, a_{6}, b_{5}$ and $b_{6}$ are obtained from the solution of the system of Eq. (A.5) and using Eqs. (A.8) and (A.10), see Appendix A.3.

\subsection{Generalized interface-enhanced Mori-Tanaka approach}

In this section we elaborate our second methodology which furnishes the global interaction (dilute concentration) tensors for the of system inhomogeneity + interface in a particulate composite. The idea of providing interaction tensors is motivated by similar techniques in the literature for coated particles or fibers (Benveniste et al., 1989; Wang et al., 2016a; 2016b; 2018). Our proposed method is similar to (Duan et al., 2007; Gu et al., 2014), but instead of looking for an equivalent particle, we seek global strain and stress tensors of the inhomogeneity/interface system. Therefore, not only can we determine the overall properties of a composite but also we will be able to determine the state of the stress and strain in each phase of the medium including the interface. This provides a significant insight towards understanding of the behavior of composites.

In Fig. 5 (left), the inhomogeneity with the elasticity modulus $L^{(1)}$ occupies the space $\Omega_{1}$ surrounded by the imperfect interface $\mathcal{I}$ and is embedded in an infinite matrix with the elasticity tensor $\boldsymbol{L}^{(2)}$. The matrix occupies the space $\Omega_{2}$ and is subject to a linear displacement $\boldsymbol{u}^{0}=\boldsymbol{\varepsilon}^{0} \cdot \boldsymbol{x}$ at far field $\partial \Omega^{\infty}$, see Chatzigeorgiou et al. (2017) for further details. Gu et al. (2014), based on the methodology developed by Duan et al. (2007), proposed to substitute the inhomogeneity + interface system with an equivalent particle, using energy principles. Instead of that methodology, here we propose to determine the dilute

(finite displacement at $r=0$ )

(traction average at $r=r_{1}$ )

(traction average at $r=r_{1}$ )

(traction equilibrium at $r=r_{1}$ )

(traction equilibrium at $r=r_{1}$ )

(traction equilibrium at $r=r_{1}$ )

(displacement continuity at $r=r_{2}$ )

strain concentration tensor and dilute stress-strain concentration tensor for the same system according to the relations

$$
\begin{aligned}
\langle\boldsymbol{\varepsilon}\rangle_{\Omega_{1}}^{+} & =\boldsymbol{T}: \boldsymbol{\varepsilon}^{0}=\frac{1}{2\left|\Omega_{1}\right|} \int_{I}\left[\boldsymbol{u}^{+} \otimes \overline{\boldsymbol{n}}+\overline{\boldsymbol{n}} \otimes \boldsymbol{u}^{+}\right] \mathrm{d} A \quad \text { and } \quad\langle\boldsymbol{\sigma}\rangle_{\Omega_{1}}^{+} \\
& =\boldsymbol{H}: \boldsymbol{\varepsilon}^{0}=\frac{1}{\left|\Omega_{1}\right|} \int_{\Omega_{1}} \sigma^{-} \mathrm{d} V+\frac{1}{\left|\Omega_{1}\right|} \int_{I} \bar{\sigma} \mathrm{d} A
\end{aligned}
$$

Also, one can identify the pure particle concentration tensor as

$\langle\boldsymbol{\varepsilon}\rangle_{\Omega_{1}}^{-}=\boldsymbol{T}^{(1)} \cdot \boldsymbol{\varepsilon}^{0}=\frac{1}{2\left|\Omega_{1}\right|} \int_{I}\left[\boldsymbol{u}^{-} \otimes \overline{\boldsymbol{n}}+\overline{\boldsymbol{n}} \otimes \boldsymbol{u}^{-}\right] \mathrm{d} A$.

Now, consider an RVE that occupies the space $\mathcal{B}$ with the boundary $\partial \mathcal{B}$ and the volume $\mathscr{V}$ subject to macroscopic strain $\mathbb{M}_{\varepsilon}$ as shown in Fig. 5 (right). The inhomogeneity occupies the space $\mathcal{B}_{1}$ with volume $\mathscr{V}_{1}$ and the matrix occupies the space $\mathcal{B}_{2}$ with volume $\mathscr{V}_{2}$. Obviously, we have $\mathcal{B}=\mathcal{B}_{1} \cup \mathcal{B}_{2}$ and $\mathscr{V}=\mathscr{V}_{1}+\mathscr{V}_{2}$ and the inhomogeneity volume fraction reads $f=\mathscr{V}_{1} / \mathscr{V}$. The macroscopic strain and stress tensors are related to the corresponding fields in the inhomogeneity and the interface according to 


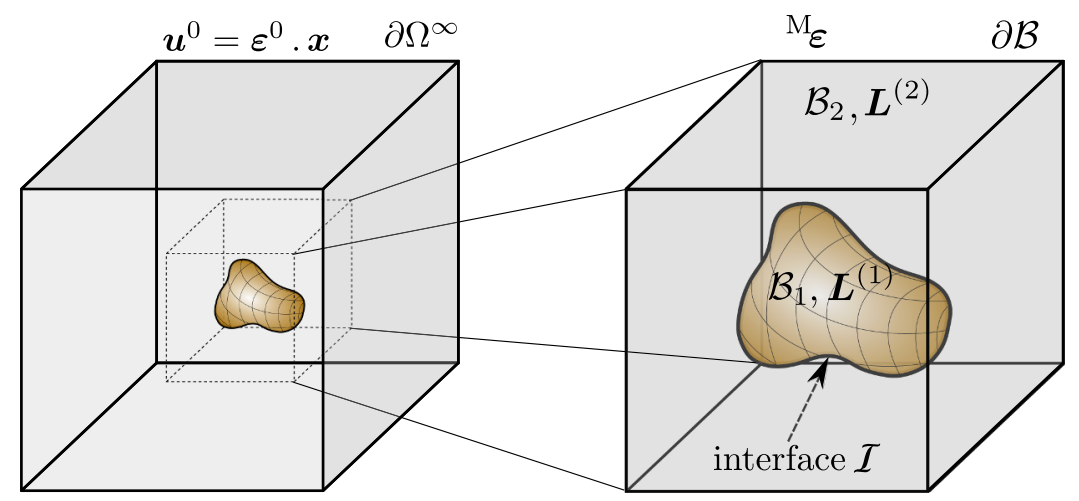

Fig. 5. Inhomogeneity with imperfect interface inside an infinite matrix (left) and the RVE consisting of the inhomogeneity with imperfect interface inside a matrix material (right).

$$
\begin{aligned}
\mathrm{M}_{\boldsymbol{\varepsilon}}= & \frac{1}{\mathscr{V}} \int_{\mathcal{B}} \boldsymbol{\varepsilon} \mathrm{d} V+\frac{1}{2 \mathscr{V}} \int_{I}[[[\boldsymbol{u}]] \otimes \overline{\boldsymbol{n}}+\overline{\boldsymbol{n}} \otimes[[\boldsymbol{u}]]] \mathrm{d} A \\
& =[1-f] \boldsymbol{\varepsilon}^{(2)}+f \boldsymbol{\varepsilon}^{(1)}+\hat{\boldsymbol{\varepsilon}}, \\
\mathrm{M}_{\boldsymbol{\sigma}}= & \left.\frac{1}{\mathscr{V}} \int_{\mathcal{B}} \sigma \mathrm{d} V+\frac{1}{\mathscr{V}} \int_{I} \bar{\sigma} \mathrm{d} A=[1-f] \boldsymbol{L}^{(2)}: \boldsymbol{\varepsilon}^{(2)}+f \boldsymbol{L}^{(1)}: \boldsymbol{\varepsilon}^{(1)}+\hat{\mathscr{4}}_{2}\right)
\end{aligned}
$$

where

$$
\begin{aligned}
\boldsymbol{\varepsilon}^{(1)} & =\frac{1}{\mathscr{V} 1} \int_{\mathcal{B}_{1}} \boldsymbol{\varepsilon} \mathrm{d} V, \quad \boldsymbol{\varepsilon}^{(2)}=\frac{1}{\mathscr{V}_{2}} \int_{\mathcal{B}_{2}} \boldsymbol{\varepsilon} \mathrm{d} V \quad \text { and } \quad \hat{\boldsymbol{\varepsilon}} \\
& =\frac{1}{2 \mathscr{V}} \int_{I}[[[\boldsymbol{u}]] \otimes \overline{\boldsymbol{n}}+\overline{\boldsymbol{n}} \otimes[[\boldsymbol{u}]]] \mathrm{d} A,
\end{aligned}
$$

are the average strains of the matrix, inhomogeneity and interface, respectively. The average stress on the interface reads

$\hat{\sigma}=\frac{1}{\mathscr{V}} \int_{I} \bar{\sigma} \mathrm{d} A$.

Utilizing the interaction tensors (40) and (41), the interface-enhanced Mori-Tanaka scheme results in

$\boldsymbol{\varepsilon}^{(1)}=\boldsymbol{T}^{(1)}: \boldsymbol{\varepsilon}^{(2)}, \quad \boldsymbol{\varepsilon}^{(1)}+\frac{1}{f} \hat{\boldsymbol{\varepsilon}}=\boldsymbol{T}: \boldsymbol{\varepsilon}^{(2)}, \quad \boldsymbol{L}^{(1)}: \boldsymbol{\varepsilon}^{(1)}+\frac{1}{f} \hat{\boldsymbol{\sigma}}=\boldsymbol{H}: \boldsymbol{\varepsilon}^{(2)}$.

Thus, Eq. (42) 1 yields

$\mathrm{M}_{\boldsymbol{\varepsilon}}=[[1-f] \llbracket+f \boldsymbol{T}] \boldsymbol{\varepsilon}^{(2)} \quad$ or $\quad \boldsymbol{\varepsilon}^{(2)}=\boldsymbol{A}^{(2)}: \mathrm{M}_{\boldsymbol{\varepsilon}}$,

with I being the fourth order identity tensor and $\boldsymbol{A}^{(2)}=[[1-f] \rrbracket+f \boldsymbol{T}]^{-1}$. On the other hand, Eq. (42) 2 yields

$\mathrm{M}_{\boldsymbol{\sigma}}=\left[[1-f] \boldsymbol{L}^{(2)}+f \boldsymbol{H}\right]: \boldsymbol{\varepsilon}^{(2)}=\left[[1-f] \boldsymbol{L}^{(2)}+f \boldsymbol{H}\right]: \boldsymbol{A}^{(2)}: \mathrm{M}_{\boldsymbol{\varepsilon}}$.

Accordingly, the macroscopic stiffness tensor reads

$\mathrm{M}_{\mathbf{L}}=\left[[1-f] \boldsymbol{L}^{(2)}+f \boldsymbol{H}\right]: \boldsymbol{A}^{(2)}$,

and the properties of the equivalent particle in (Gu et al., 2014) can be recovered according to $\boldsymbol{L}^{\mathrm{eq}}=\boldsymbol{H}: \boldsymbol{T}^{-1}$. For a given macroscopic strain $\mathrm{M}_{\boldsymbol{\varepsilon}}$, the average stress and strain in the particle and the matrix are

$\sigma^{(1)}=\boldsymbol{L}^{(1)}: \boldsymbol{\varepsilon}^{(1)}=\boldsymbol{L}^{(1)}: \boldsymbol{T}^{(1)}: \boldsymbol{A}^{(2)}: \mathrm{M}_{\boldsymbol{\varepsilon}}, \quad \boldsymbol{\varepsilon}^{(1)}=\boldsymbol{T}^{(1)}: \boldsymbol{A}^{(2)}: \mathrm{M}_{\boldsymbol{\varepsilon}}$,

$\sigma^{(2)}=\boldsymbol{L}^{(2)}: \boldsymbol{\varepsilon}^{(2)}=\boldsymbol{L}^{(2)}: \boldsymbol{A}^{(2)}: \mathrm{M}_{\boldsymbol{\varepsilon}}, \quad \boldsymbol{\varepsilon}^{(2)}=\boldsymbol{A}^{(2)}: \mathrm{M}_{\boldsymbol{\varepsilon}}$.

Exploiting Eq. (45), the average stress and strain on the interface are given by

$\hat{\boldsymbol{\sigma}}=f\left[\boldsymbol{H}-\boldsymbol{L}^{(1)}: \boldsymbol{T}^{(1)}\right]: \boldsymbol{A}^{(2)}: \mathrm{M}_{\boldsymbol{\varepsilon}} \quad$ and $\quad \hat{\boldsymbol{\varepsilon}}=f\left[\boldsymbol{T}-\boldsymbol{T}^{(1)}\right]: \boldsymbol{A}^{(2)}: \mathrm{M}_{\boldsymbol{\varepsilon}}$.

In order to complete the homogenization framework, we must determine the interaction tensors $\boldsymbol{H}, \boldsymbol{T}$ and $\boldsymbol{T}^{(1)}$. In doing so, we employ the Eshelby's inhomogeneity problem for isochoric and deviatoric conditions. For a particle reinforced composite with isotropic constituents, the interaction tensors are isotropic and can be written as

$$
\begin{aligned}
& \boldsymbol{T}^{(1)}=3 T^{b(1)} \mathcal{I}^{h}+2 T^{s(1)} \mathcal{I}^{d}, \quad \boldsymbol{T}=3 T^{b} \mathcal{I}^{h}+2 T^{s} \mathcal{I}^{d}, \\
& \boldsymbol{H}=3 H^{b} \mathcal{I}^{h}+2 H^{s} \mathcal{I}^{d} \quad \text { with } \quad \mathcal{I}^{h}=\frac{1}{3} \boldsymbol{i} \otimes \boldsymbol{i}, \quad \mathcal{I}^{d}=\mathcal{I}-\mathcal{I}^{h} .
\end{aligned}
$$

\subsubsection{Isochoric conditions}

Assume an RVE consisting of an infinite matrix with a spherical inhomogeneity subject to a hydrostatic far field displacement according to

$\boldsymbol{u}_{(x, y, z)}^{0}=\left[\begin{array}{c}\beta x \\ \beta y \\ \beta z\end{array}\right]$ and $\boldsymbol{u}_{(r, \theta, \phi)}^{0}=\left[\begin{array}{c}\beta r \\ 0 \\ 0\end{array}\right]$.

For this boundary condition, the displacement fields in the matrix and fiber are similar to Eq. (18) with the unknowns $\Xi_{1}^{(1)}, \Xi_{2}^{(1)}, \Xi_{1}^{(2)}$ and $\Xi_{2}^{(2)}$. The boundary and interface conditions lead to the following equations

$$
\begin{aligned}
& u_{r}^{(1)} \text { finite at } r=0 \rightarrow \Xi_{2}^{(1)}=0, \\
& \bar{t}_{r}=\bar{k}_{r}\left[\left[u_{r}\right]\right] \quad \rightarrow \frac{\sigma_{r r}^{(2)}\left(r_{1}\right)+\sigma_{r r}^{(1)}\left(r_{1}\right)}{2} \\
& =\bar{k}_{r}\left[u_{r}^{(2)}\left(r_{1}\right)-u_{r}^{(1)}\left(r_{1}\right)\right] \text {, } \\
& {[\overline{\operatorname{div}} \bar{\sigma}]_{r}+\left[\left[t_{r}\right]\right]=0 \rightarrow-\frac{\bar{\sigma}_{\theta \theta}+\bar{\sigma}_{\phi \phi}}{r_{1}}+\sigma_{r r}^{(2)}\left(r_{1}\right)-\sigma_{r r}^{(1)}\left(r_{1}\right) \text { (traction equilibrium at } r=r_{1} \text { ) }} \\
& \begin{array}{c}
=0, \\
u_{r}^{(2)}(r \rightarrow \infty)=\beta r, \quad \rightarrow \quad \Xi_{1}^{(2)}=1,
\end{array} \\
& \text { (finite displacement at } r=0 \text { ) } \\
& \text { (traction average at } r=r_{1} \text { ) } \\
& \text { (displacement at } r \rightarrow \infty \text { ) }
\end{aligned}
$$

resulting in the linear system of equations

$\left[\begin{array}{cc}1+\frac{3 \kappa_{1}}{2 \bar{k} r_{1}} & -1-\frac{2 \mu_{2}}{\bar{k} r_{1}} \\ 3 \kappa_{1}+\frac{2 \bar{k}}{r_{1}} & 4 \mu_{2}+\frac{2[\bar{\lambda}+\bar{\mu}]}{r_{1}}\end{array}\right]\left[\begin{array}{l}\Xi_{1}^{(1)} \\ \Xi_{2}^{(2)}\end{array}\right]=\left[\begin{array}{c}1-\frac{3 \kappa_{2}}{2 \bar{k} r_{1}} \\ 3 \kappa_{2}-\frac{2[\bar{\lambda}+\bar{\mu}]}{r_{1}}\end{array}\right]$.

Solving this system, the average strain in the particle and the strain and stress fields in the particle + interface system are obtained as

$$
\begin{gathered}
\int_{\mathcal{B}} \varepsilon_{\Omega_{1}}^{-} \mathrm{d} V=\Xi_{1}^{(1)} \varepsilon^{0}, \quad \int_{\mathcal{B}} \varepsilon_{\Omega_{1}}^{+} \mathrm{d} V=\left[1+\Xi_{2}^{(2)}\right] \boldsymbol{\varepsilon}^{0}, \\
\int_{\mathcal{B}} \sigma_{\Omega_{1}}^{+} \mathrm{d} V=\left[3 \kappa_{2}-4 \mu_{2} \Xi_{2}^{(2)}\right] \boldsymbol{\varepsilon}^{0} .
\end{gathered}
$$

Consequently, the bulk interaction terms read

$3 T^{b(1)}=\Xi_{1}^{(1)}, \quad 3 T^{b}=1+\Xi_{2}^{(2)}, \quad 3 H^{b}=3 \kappa_{2}-4 \mu_{2} \Xi_{2}^{(2)}$,

where $\Xi_{1}^{(1)}$ and $\Xi_{2}^{(2)}$ are obtained from the solution of the linear system (54). 


\subsubsection{Deviatoric conditions}

Assume the RVE is subject to a deviatoric far field displacement

$\boldsymbol{u}_{(x, y, z)}^{0}=\left[\begin{array}{c}\beta y \\ -\beta x \\ 0\end{array}\right]$ and $u_{(r, \theta, \phi)}^{0}=\left[\begin{array}{c}\beta r \sin ^{2} \theta \cos 2 \phi \\ \beta r \sin \theta \cos \theta \cos 2 \phi \\ -\beta r \sin \theta \sin 2 \phi\end{array}\right]$.

For this boundary condition, the displacement fields in the matrix and fiber are similar to Eq. (24) with the unknowns $\Xi_{1}^{(1)}, \Xi_{2}^{(1)}, \Xi_{3}^{(1)}, \Xi_{4}^{(1)}, \Xi_{1}^{(2)}$, $\Xi_{2}^{(2)}, \Xi_{3}^{(2)}$ and $\Xi_{4}^{(2)}$. The boundary and interface conditions lead to the following equations
Solving this system, the average strain in the particle and the strain and stress in the particle + interface system are determined as

$$
\begin{aligned}
& \int_{\mathcal{B}} \varepsilon_{\Omega_{1}}^{-} \mathrm{d} V=\frac{1}{5}\left[5 \Xi_{1}^{(1)}-7\left[1+3 \frac{\varkappa_{1}}{\mu_{1}}\right] \Xi_{2}^{(1)}\right] \boldsymbol{\varepsilon}^{0}, \\
& \int_{\mathcal{B}} \varepsilon_{\Omega_{1}}^{+} \mathrm{d} V=\frac{1}{5}\left[5+6\left[2+\frac{\kappa_{2}}{\mu_{2}}\right] \Xi_{4}^{(2)}\right] \boldsymbol{\varepsilon}^{0}, \\
& \int_{\mathcal{B}} \sigma_{\Omega_{1}}^{+} \mathrm{d} V=\frac{1}{5}\left[10 \mu_{2}-2\left[9 \kappa_{2}+8 \mu_{2}\right] \Xi_{4}^{(2)}\right] \boldsymbol{\varepsilon}^{0} .
\end{aligned}
$$

Consequently, the shear interaction terms read

$$
\begin{aligned}
& u_{r}^{(1)}, u_{\theta}^{(1)} \text { finite at } r=0 \quad \rightarrow \Xi_{3}^{(1)}=\Xi_{4}^{(1)}=0, \\
& \text { finite displacement at } r=0 \\
& \bar{t}_{r}=\bar{k}_{r}\left[\left[u_{r}\right]\right] \\
& \rightarrow \sigma_{r r}^{(2)}\left(r_{1}\right)+\sigma_{r r}^{(1)}\left(r_{1}\right)=2 \bar{k}_{r}\left[u_{r}^{(2)}\left(r_{1}\right)-u_{r}^{(1)}\left(r_{1}\right)\right], \quad\left(\text { traction average at } r=r_{1}\right. \text { ) } \\
& \bar{t}_{\theta}=\bar{k}_{\theta}\left[\left[u_{\theta}\right]\right] \\
& \rightarrow \sigma_{r \theta}^{(2)}\left(r_{1}\right)+\sigma_{r \theta}^{(1)}\left(r_{1}\right)=2 \bar{k}_{\theta}\left[u_{\theta}^{(2)}\left(r_{1}\right)-u_{\theta}^{(1)}\left(r_{1}\right)\right] \text {, (traction average at } r=r_{1} \text { ) } \\
& {[\overline{\operatorname{div}} \bar{\sigma}]_{r}+\left[\left[t_{r}\right]\right]=0} \\
& \rightarrow-\frac{\bar{\sigma}_{\theta \theta}+\bar{\sigma}_{\phi \phi}}{r_{1}}+\sigma_{r r}^{(2)}\left(r_{1}\right)-\sigma_{r r}^{(1)}\left(r_{1}\right)=0 \text {, } \\
& \text { (traction equilibrium at } r=r_{1} \text { ) } \\
& \rightarrow \frac{1}{r_{1}} \frac{\partial \bar{\sigma}_{\theta \theta}}{\partial \theta}+\frac{1}{r_{1} \sin \theta} \frac{\partial \bar{\sigma}_{\theta \phi}}{\partial \phi}+\frac{\left[\bar{\sigma}_{\theta \theta}-\bar{\sigma}_{\phi \phi}\right] \cos \theta}{r_{1} \sin \theta} \\
& +\sigma_{r \theta}^{(2)}\left(r_{1}\right)-\sigma_{r \theta}^{(1)}\left(r_{1}\right)=0, \\
& \rightarrow \Xi_{1}^{(2)}=1, \quad \Xi_{2}^{(2)}=0,
\end{aligned}
$$

resulting in the system of equations

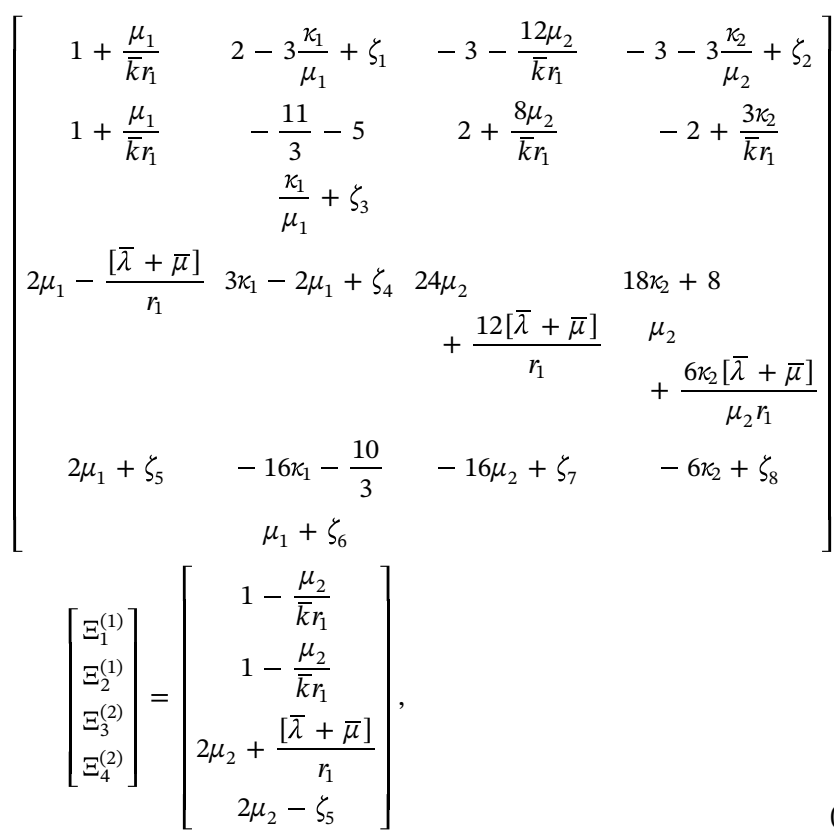

with

$$
\begin{aligned}
& \zeta_{1}=\frac{3 \kappa_{1}-2 \mu_{1}}{2 \bar{k} r_{1}}, \quad \zeta_{2}=-\frac{9 \kappa_{2}+4 \mu_{2}}{\bar{k} r_{1}}, \quad \zeta_{3}=-\frac{24 \kappa_{1}+5 \mu_{1}}{3 \bar{k} r_{1}}, \\
& \zeta_{4}=\frac{\left[9 \kappa_{1}+15 \mu_{1}\right][\bar{\lambda}+\bar{\mu}]}{\mu_{1} r_{1}}, \quad \zeta_{5}=\frac{[\bar{\lambda}+3 \bar{\mu}]}{r_{1}}, \\
& \zeta_{6}=-\frac{\varkappa_{1}[9 \bar{\lambda}+19 \bar{\mu}]}{\mu_{1} r_{1}}-\frac{[45 \bar{\lambda}+67 \bar{\mu}]}{3 r_{1}}, \quad \zeta_{7}=-\frac{4[3 \bar{\lambda}+4 \bar{\mu}]}{r_{1}}, \\
& \zeta_{8}=\frac{-6 \kappa_{2}[\bar{\lambda}+\bar{\mu}]+4 \mu_{2} \bar{\mu}}{\mu_{2} r_{1}} .
\end{aligned}
$$

$$
\begin{aligned}
& 2 T^{s(1)}=\frac{1}{5}\left[5 \Xi_{1}^{(1)}-7\left[1+3 \frac{\varkappa_{1}}{\mu_{1}}\right] \Xi_{2}^{(1)}\right], \\
& 2 T^{s}=\frac{1}{5}\left[5+6\left[2+\frac{\varkappa_{2}}{\mu_{2}}\right] \Xi_{4}^{(2)}\right], \quad 2 H^{s}=\frac{1}{5}\left[10 \mu_{2}-2\left[9 \varkappa_{2}+8 \mu_{2}\right] \Xi_{4}^{(2)}\right] .
\end{aligned}
$$

where $\Xi_{1}^{(1)}, \Xi_{2}^{(1)}$ and $\Xi_{4}^{(2)}$ are obtained from the linear system (59).

Remark. The composite spheres assemblage methodology has been designed for uniform distribution of inclusions and is not extensible to more complex cases. On the other hand, the Mori-Tanaka approach is more flexible and non-uniform distributions of inclusions can be adopted by proper modification of the relations (40) and (41), see for instance (Entchev and Lagoudas, 2002). The relation between the tensors $\boldsymbol{T}$ and $\boldsymbol{A}$ provides implicitly the interactions between inclusions of different type or distribution.

\section{Numerical examples}

In this section, through a set of numerical examples, the accuracy of the analytical solutions are evaluated via comparison with the computational results using the finite element method. In doing so, the overall material response of particulate composites embedding general interfaces is investigated in various scenarios. It shall be emphasized that the results obtained by the computational analysis are regarded as the "exact" solution and the analytical solutions are interpreted as "approximations". The RVE in our computational study is spherical suitable for comparison with the analytical solutions. The computational analysis is carried out using our in-house finite element code applied to the RVE discretized by quadratic Lagrange elements as depicted in Fig. 6. The discretized RVE consists of overall 19208 elements and 160863 nodes. For all the examples, the solution procedures are robust and render asymptotically quadratic rate of convergence associated with the Newton-Raphson scheme. Throughout the examples, the inclusion volume fraction is assumed to be $30 \%$. To cover a broad 


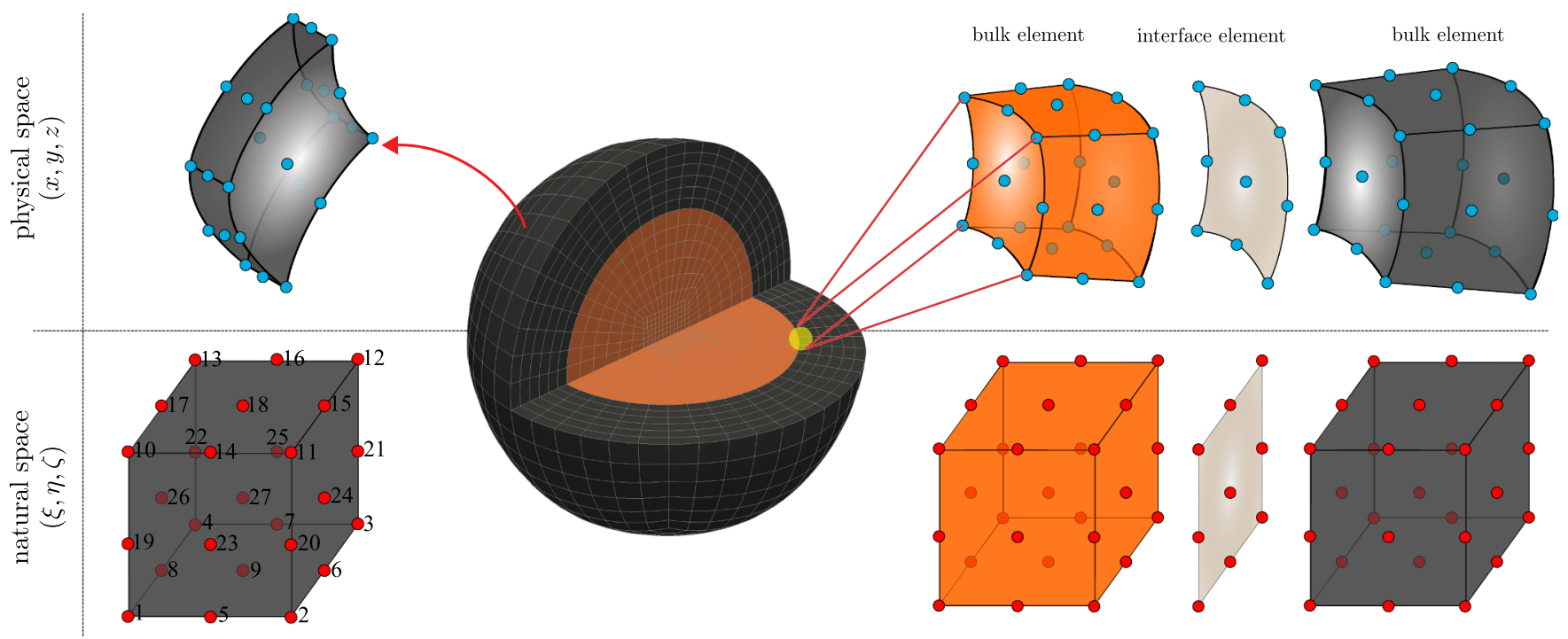

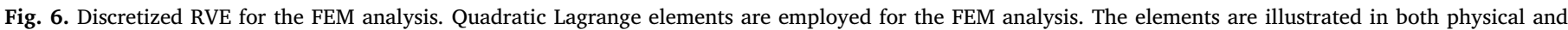
natural spaces.

range of material properties, three different stiffness ratios of $0.1,1$ and 10 are examined. The stiffness ratio denoted as incl./matr. is the ratio of the inclusion Lamé parameters to the matrix Lamé parameters. The stiffness ratio 0.1 indicates a 10 times more compliant inclusion compared to the matrix whereas the stiffness ratio 10 corresponds to a 10 times stiffer inclusion than the matrix. The stiffness ratio 1 represents identical inclusion and matrix. Obviously, the limit case of incl./ matr. $\rightarrow \infty$ indicates a rigid inclusion whereas incl. $/$ matr. $=0$ corresponds to porous media. In this study, we set the matrix material parameters to $\lambda_{2}=\mu_{2}=1$ and the inclusion material parameters vary in accordance with the predefined stiffness ratios. In order to highlight the role of the general interface in the overall material response, two values of $\bar{\lambda}=\bar{\mu}=1$ and $\bar{\lambda}=\bar{\mu}=100$ are considered for the general interface in-plane parameters indicating a low and a high elastic resistance against in-plane stretches, respectively. On the other hand, the two considered values for the general interface orthogonal resistance against opening are $\bar{k}=1$ indicating a low stiffness and $\bar{k}=100$ indicating a high orthogonal resistance. In the limit of $\bar{k}=0$, the interface shows no opening resistance resembling a totally detached matrix and particle. In contrast, the limit of $\bar{k} \rightarrow \infty$ corresponds to a coherent interface. We emphasize that our developed general interface model is capable to recover any of the cohesive, elastic or perfect interface models, see Fig. 1. The elastic interface model is recovered when $\bar{\lambda} \neq 0$, $\bar{\mu} \neq 0$ and $\bar{k} \rightarrow \infty$. The conditions $\bar{\lambda}=0$ and $\bar{\mu}=0$ recover the cohesive interface model with coherent coefficient $\bar{k}$. Finally, the perfect interface model can be considered as the coincidence of the cohesive and the elastic interface models with $\bar{\lambda}=0, \bar{\mu}=0$ and $\bar{k} \rightarrow \infty$.

Figs. 7 -9 show the effective bulk and shear moduli ${ }^{M_{k}}$ and ${ }^{M_{\mu}}$ versus size for different stiffness ratios. Each row corresponds to a specific interface orthogonal resistance and each column corresponds to a specific interface in-plane resistance. Clearly, the first column represents the cohesive interface model due to the vanishing interface inplane resistance. The dashed lines show the solutions obtained from the analytical approaches developed in Sections 3.2 and 3.3. The solid straight line shows the response associated with the perfect interface model. The red circular points and blue rectangular points correspond to the computational results via imposing $\mathrm{DBC}$ and $\mathrm{TBC}$, respectively.

A remarkable agreement is consistently obtained between the analytical and computational results. For all cases, size-dependent behavior is observed due to the presence of the interface. For the bulk modulus, all the solutions show a uniform behavior relative to the perfect interface solution. The results coincide with the perfect interface model at small sizes. As the size increases, the overall response deviates from the perfect interface solution until it reaches to an extermum at a critical size which is then followed by converging to the perfect interface solution due to negligible interface effects at large sizes. When incl. $/$ matr. $=0.1$, the results from the general interface model always render a stiffer response than that of the perfect interface model. In addition, increasing any of the interface parameters stiffens the overall response. A particularly important observation is that for incl./matr. $=1$, although the matrix and the inclusion are identical, various combinations of general interface parameters result in different, but yet size-dependent, overall behavior. If incl./matr. = 10, depending on the interface parameters, the general interface model could result in either stiffer or weaker response compared to the perfect interface model. Somewhat surprisingly, the overall response almost show no sensitivity to the size for some special cases, for instance, the cases with incl./matr. $=10, \bar{\lambda}=\bar{\mu}=1$ and $\bar{k}=100$. For the shear modulus, there is a perfect agreement between DBC and the upper bound and between TBC and the lower bound. Depending on the stiffness ratio and the general interface parameters, various observations can be drawn. For instance when incl. $/$ matr. $=0.1$, the bounds coincide only at certain sizes. Increasing any of the interface parameters yields a stiffer material response. For incl./matr. $=1$, there is an excellent agreement between the bounds at large sizes. This is justifiable since incl./matr. $=1$ implies identical matrix and inclusion and at large sizes interface effects diminish and bulk properties play the decisive role on the overall material response. If incl./matr. $=10$, the bounds agree only at few sizes for weak interfaces. Increasing any of the interface parameters widens the gap between the upper and the lower bounds. Another noteworthy observation is that the generalized self-consistent method and the modified Mori-Tanaka method do not provide similar estimates for the macroscopic shear modulus. It is observed that generally GSCM provides higher values than the MT method.

Remark. In view of the behavior of the effective bulk modulus $\mathrm{M}_{\mathcal{k}}$, it is observed that the general interface model at both limits of small and large sizes converges to the perfect interface model. The interface effect is decreasing when increasing the size and thus, its behavior at large sizes is fairly obvious. At small scales, however, further discussion is required to justify the influence of the interface on the overall material response. The effective behavior of the general interface model can be explained by the fact that it combines the two opposing cohesive and elastic interface models, schematically illustrated in Fig. 1. The elastic interface model results in a smaller-stronger effect in contrast to the smaller-weaker effect of the cohesive interface model. At large sizes, 


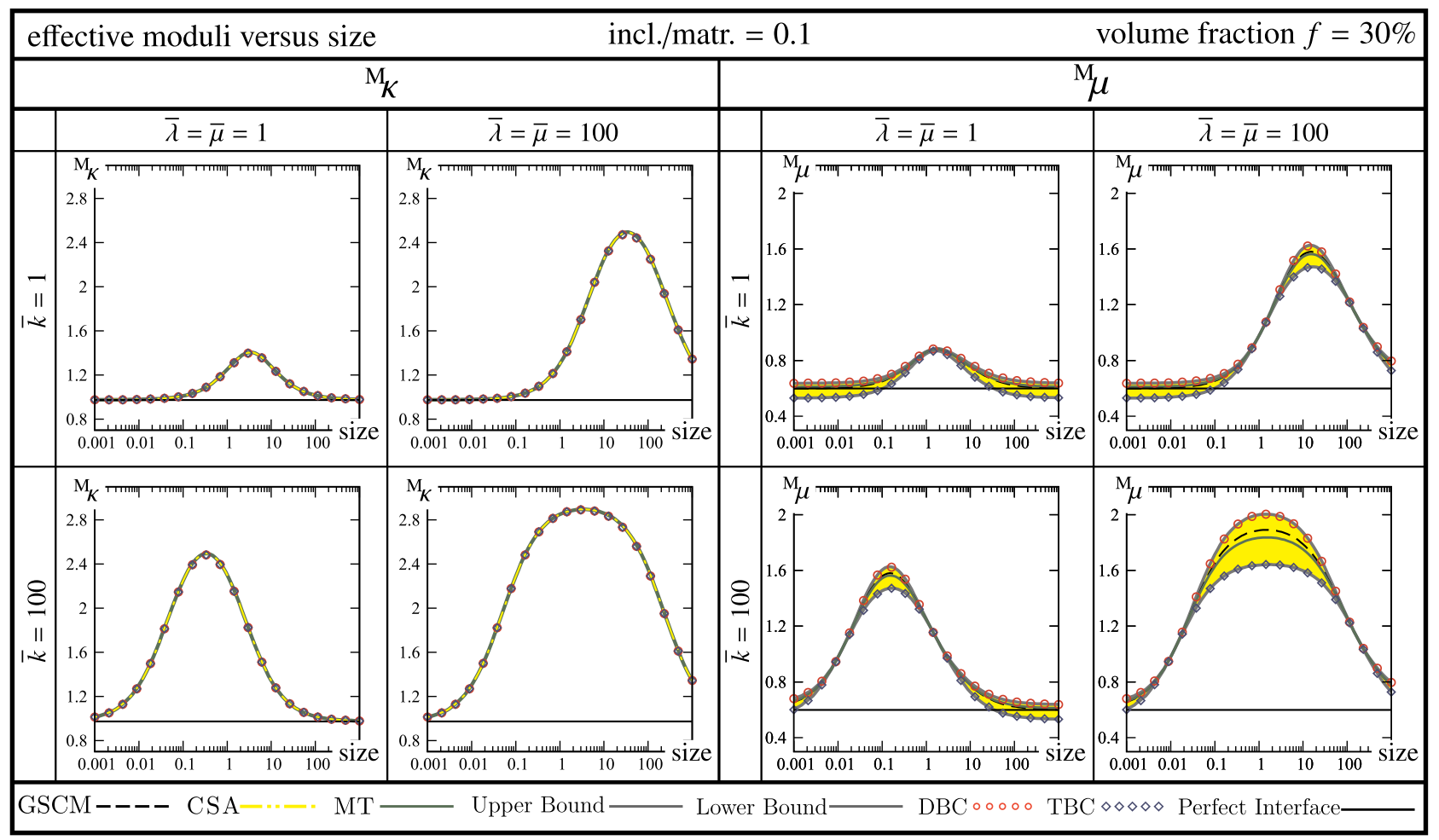

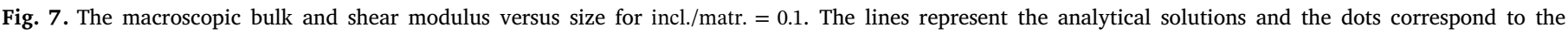

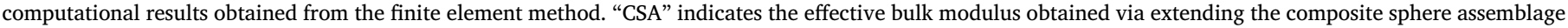

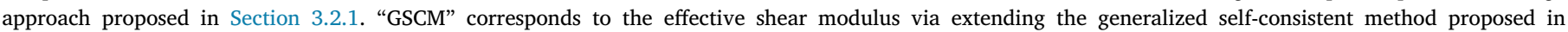

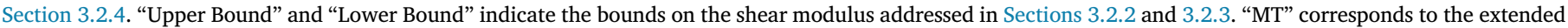
Mori-Tanaka method developed in Section 3.3.

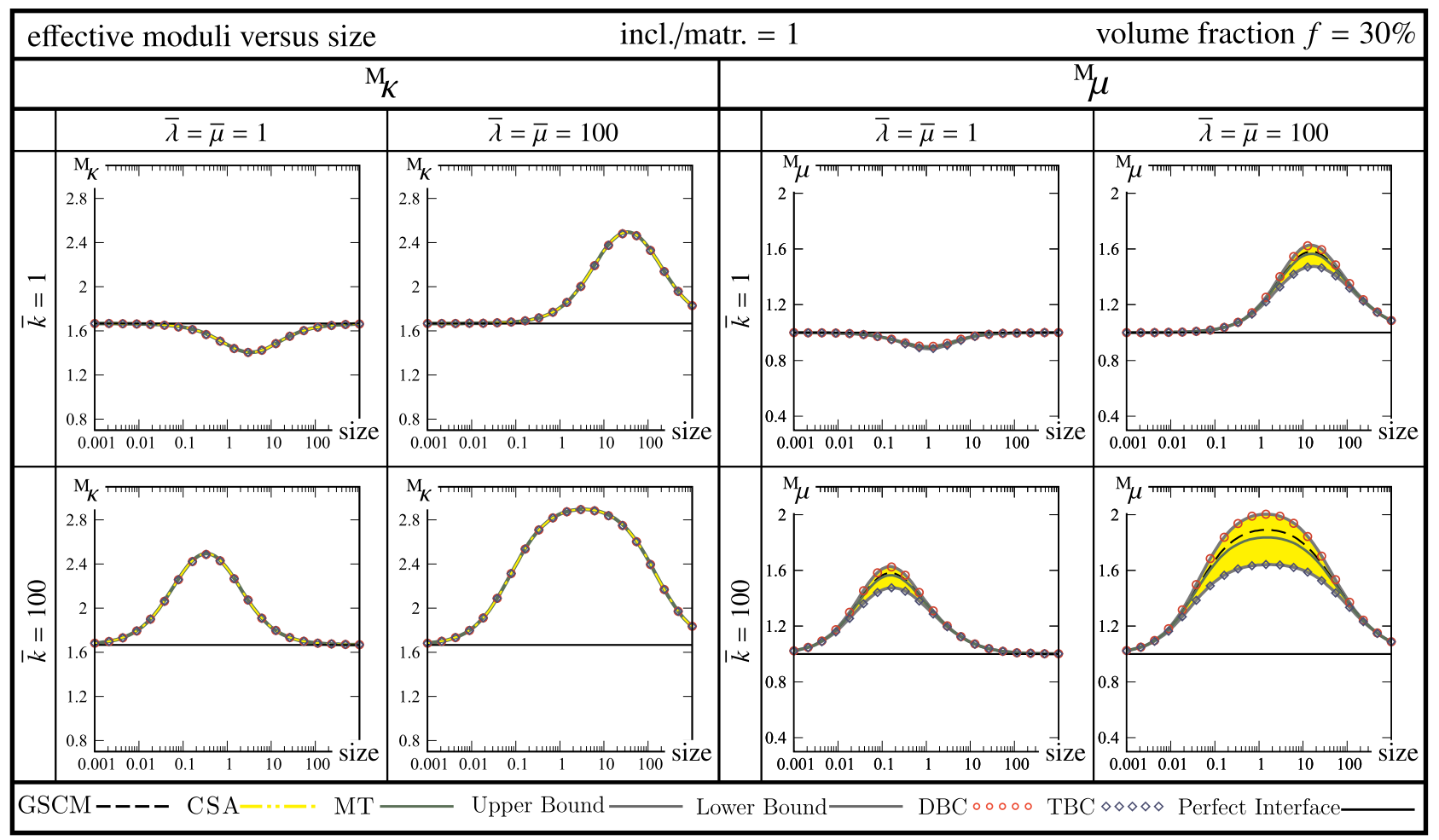

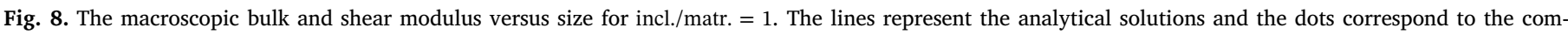

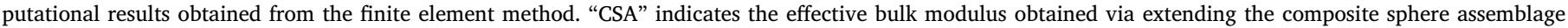

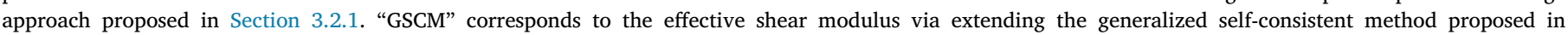

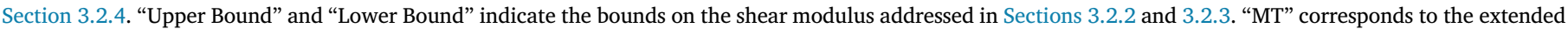
Mori-Tanaka method developed in Section 3.3. 


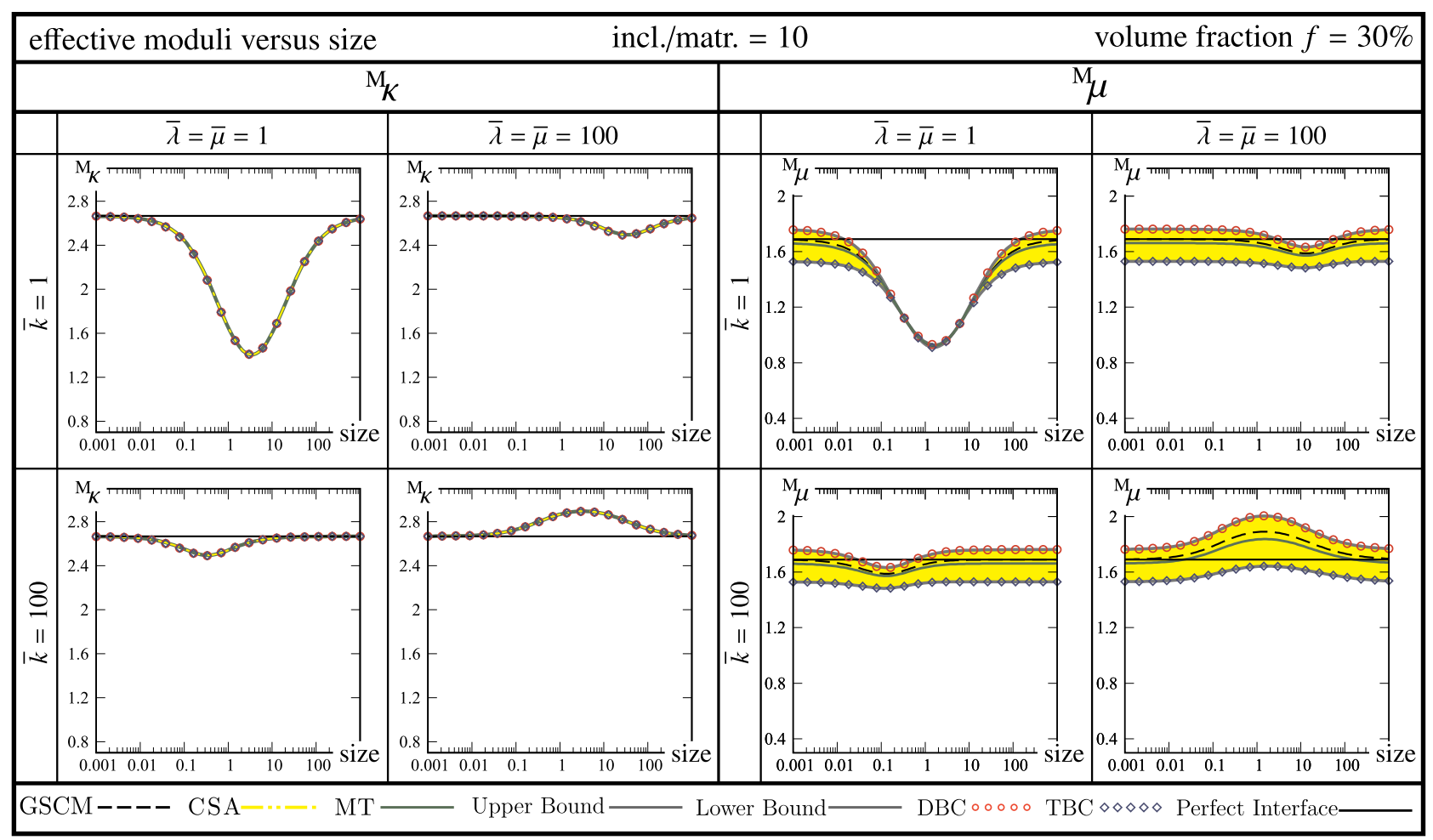

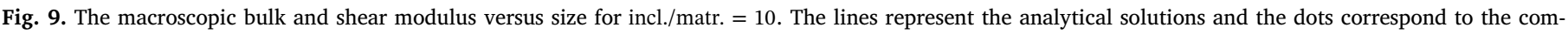

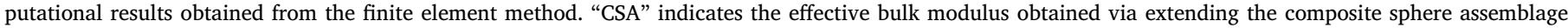

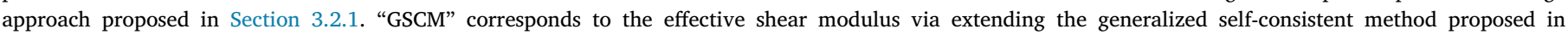

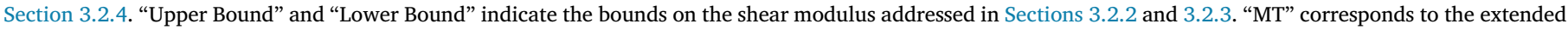
Mori-Tanaka method developed in Section 3.3.

neither of the interface effects is present. But at small sizes, both of the interface effects are present and eventually cancel each other. Furthermore, we can elaborate on this observation from an analytical perspective. To do so, we re-express the effective bulk modulus Eq. (22) as

$$
\left[\bar{\lambda}+\bar{\mu}+\bar{k} r_{1}^{2}\right]\left[3 \kappa_{1}\left[3 \kappa_{2}+4 f \mu_{2}\right]+12 \kappa_{2} \mu_{2}[1-f]\right]+4 \bar{k} r_{1}
$$

$$
\begin{aligned}
\mathrm{M}_{\kappa}=\frac{[\bar{\lambda}+\bar{\mu}]\left[3 \kappa_{2}+4 f \mu_{2}\right]+36 \kappa_{1} \kappa_{2} \mu_{2} r_{1}[1-f]}{3\left[\bar{\lambda}+\bar{\mu}+\bar{k} r_{1}^{2}\right]\left[3 \kappa_{1}+4 \mu_{2}+3 f\left[\kappa_{2}-\kappa_{1}\right]\right]+12 \bar{k} r_{1}} \\
{[\bar{\lambda}+\bar{\mu}][1-f]+9 \kappa_{1} r_{1}\left[4 \mu_{2}+3 f \kappa_{2}\right] }
\end{aligned}
$$

thereby gaining a better insight on ${ }^{M_{\mathcal{K}}}$ in terms of $r_{1}$. This relation in both limits simplifies to

$$
r \rightarrow 0 \quad \text { or } \quad r \rightarrow \infty \quad \Rightarrow \quad \mathrm{M}_{\kappa}=\kappa_{2}+\frac{f\left[\kappa_{1}-\kappa_{2}\right]\left[4 \mu_{2}+3 \kappa_{2}\right]}{4 \mu_{2}+3 \kappa_{1}+3 f\left[\kappa_{2}-\kappa_{1}\right]}
$$

which corresponds exactly to the solution associated with the perfect interface model.

Motivated by the observations throughout the examples, we can identify two distinctive size-dependent bounds and ultimate bounds, depicted in Fig. 10. Size-dependent bounds are the bounds on the overall properties of the composites at any specific size. These bounds correspond to the boundary value problem solution associated with DBC providing the upper bound and TBC providing the lower bound. On the other hand, regardless of the RVE size, the macroscopic response is always bounded between two extreme values referred to as ultimate bounds. These bounds are independent of the size and they solely depend on the interface and bulk material properties. As shown in Fig. 10, one of the ultimate bounds is reached at a critical size between the limits and the other one is reached at extreme sizes whereas size-dependent bounds are local in the sense that for specific material and interface parameters, they vary as the size changes. Note that the sizedependent bounds are only distinguishable in the case of the overall

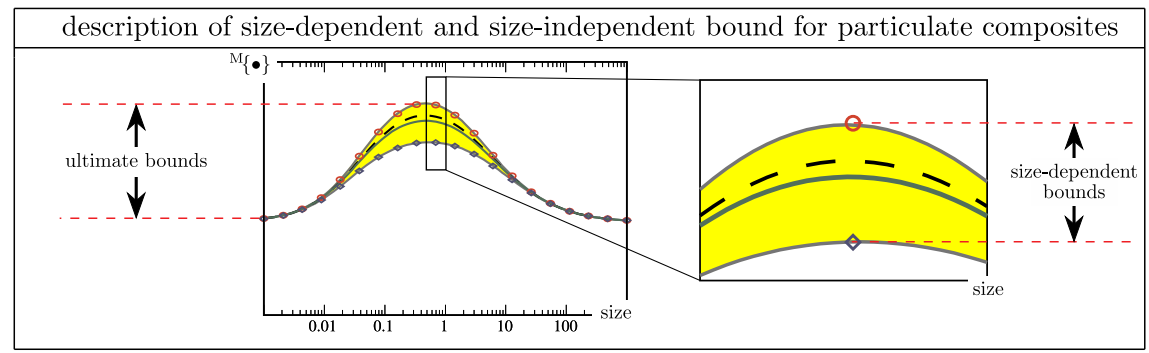

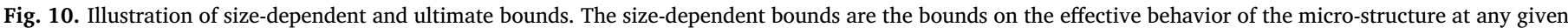
size. The ultimate bounds are size independent and entirely depend on the interface and bulk material properties. 


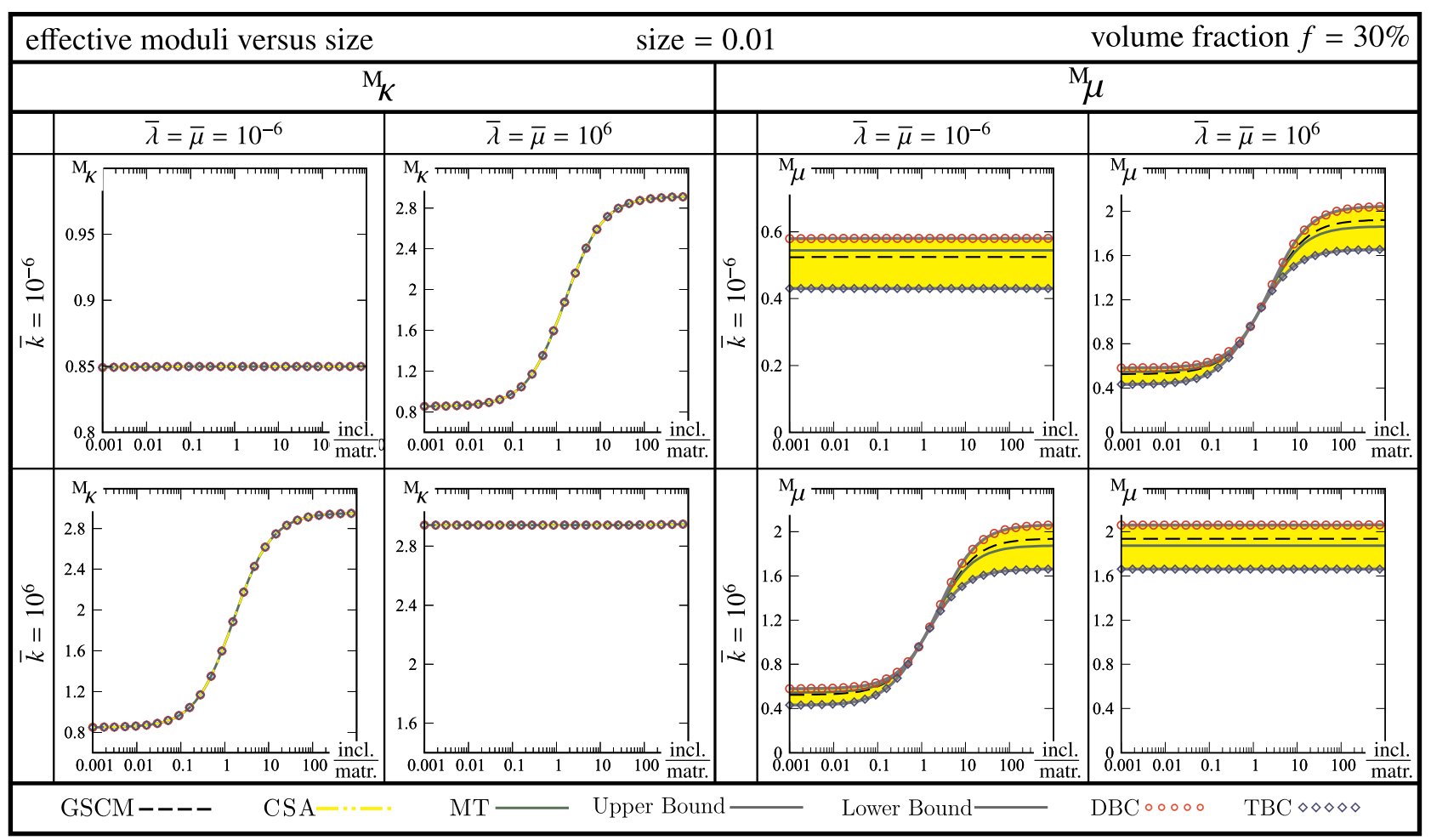

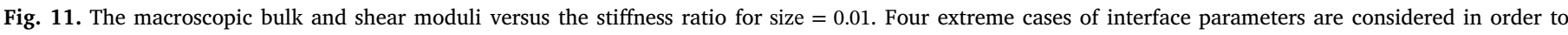
investigate the neutrality of the material response to the inclusion stiffness.

shear modulus $\mathrm{M}_{\mu}$ and they coincide in the case of the overall bulk modulus ${ }^{k_{k}}$ in accordance with the observation made by Hashin and Rosen (1964) for perfect interfaces.

So far the variation of the overall moduli versus size has been investigated for three specific stiffness ratios. In order to accentuate the role of stiffness ratio and investigate the neutrality of the overall response to the inclusion stiffness in the presence of imperfect interfaces, we study the behavior of the overall moduli versus the stiffness ratio in Fig. 11. To highlight the role of the interface, the study is carried out at a small size $=0.01$. Four extreme cases of

(i) very weak cohesive and very weak elastic interface resistance \{ $\left.\bar{k}=10^{-6}, \bar{\mu}=\bar{\lambda}=10^{-6}\right\}$,

(ii) very weak cohesive and very strong elastic interface resistance \{ $\left.\bar{k}=10^{-6}, \bar{\mu}=\bar{\lambda}=10^{6} \quad\right\}$,

(iii) very strong cohesive and very weak elastic interface resistance \{ $\left.\bar{k}=10^{6}, \bar{\mu}=\bar{\lambda}=10^{-6} \quad\right\}$,

(iv) very strong cohesive and very strong elastic interface resistance \{ $\left.\bar{k}=10^{6}, \bar{\mu}=\bar{\lambda}=10^{6}\right\}$,

for the interface parameters are considered. Note that the case (iii) in our analysis renders a response similar to that of a perfect interface model, see Fig. 1. More precisely, the general interface model in case (iii) strongly resists against opening representing a perfect bonding between the inclusion and the matrix but also, shows nearly no elastic resistance along the interface similar to the classical first-order homogenization. It is observed that in cases (i) and (iv) where both the elastic and cohesive interface responses are either very weak or very strong, the overall properties show no sensitivity with respect to the inclusion stiffness therefore, the material response becomes neutral to the inclusion. On the other hand, for the two other cases of (ii) and (iii), the overall response varies with the stiffness ratio and stiffer inclusions correspond to a stiffer overall response. The concept of neutrality of mechanical response with respect to thin fibers was presented by Goudarzi and Simone (2019) where both imperfect and perfect bondings between the fibers and matrix were assumed which shall be compared with the discussion here.

As pointed out earlier, a key feature of our proposed modified Mori-Tanaka approach is that in addition to the overall material properties, it determines the interaction tensors and consequently the stress and strain fields within each phase of the medium. The next set of examples are devised to demonstrate the utility of our modified Mori-Tanaka method to calculate the stress state in the medium subject to volumetric expansion and simple shear. Figs. 12-14 show the analytical and computational stress distributions throughout the microstructure for different sizes as well as different stiffness ratios. All of the micro-structures are schematically scaled to the same size for the sake of better illustration. In each figure, the rows correspond to specific stiffness ratios. At the left columns, volumetric expansion is applied to the micro-structure and the pressure-like quantity $\left[\sigma_{x x}+\sigma_{y y}+\sigma_{z z}\right] / 3$ is more relevant to study. On the other hand, at the right columns, simple shear in $x y$-plane is applied to the RVE in which case the stress component of interest is $[\boldsymbol{\sigma}]_{x y}$. In each box, the top micro-structures correspond to the computational local stress distributions due to DBC and TBC and the analytical stress distribution is shown at the center. Since our proposed analytical scheme determines the average stresses in the constituents of the medium, the bottom micro-structures in each box render the average computational stress due to DBC and TBC suitable for comparison with the analytical stresses. The average stresses in the particle and the matrix are stated below each case for the sake of 


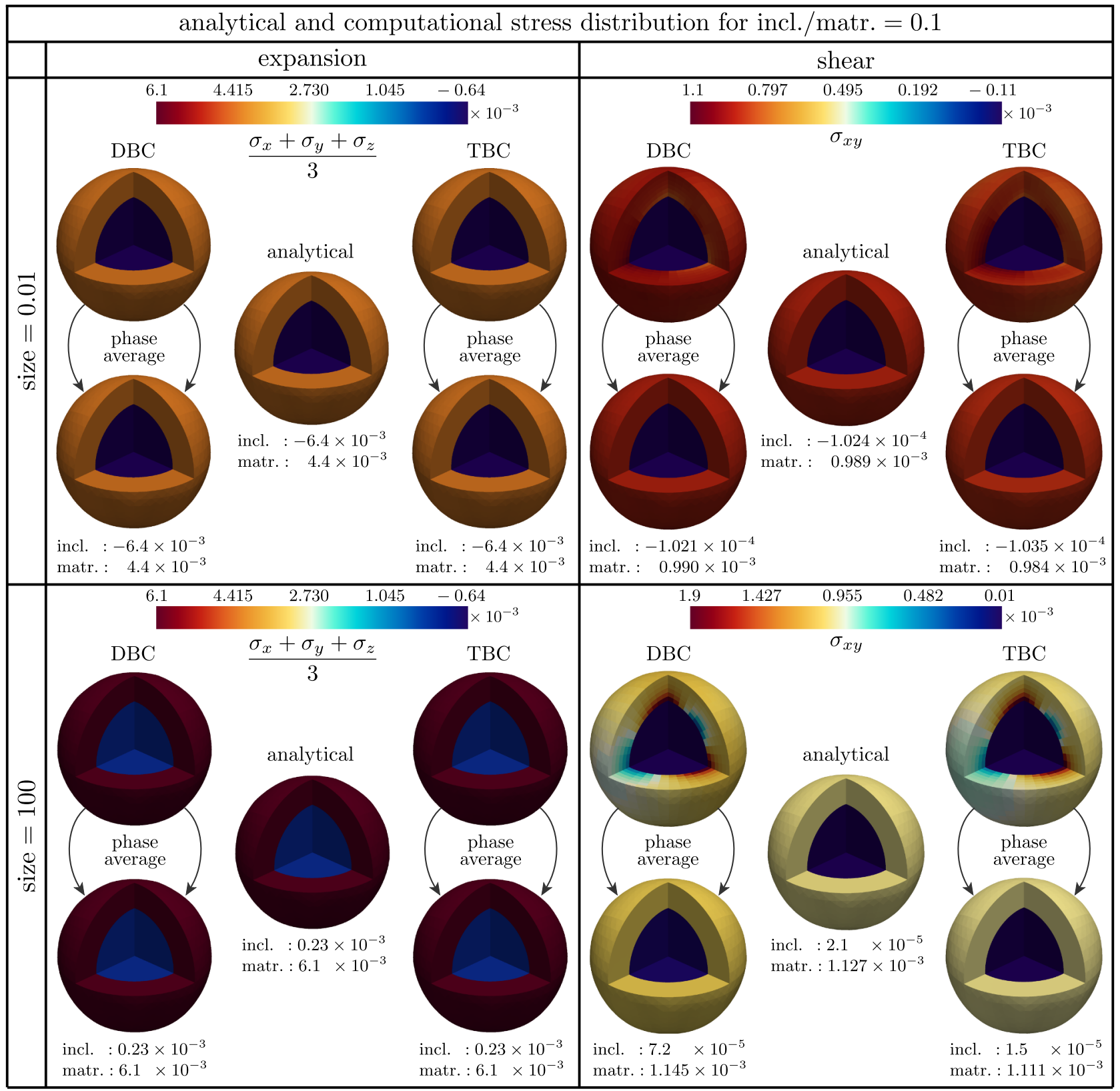

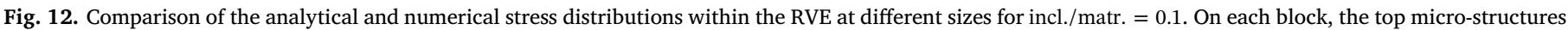

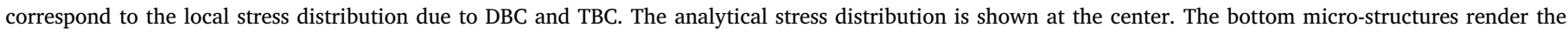
average of the computational stresses due to DBC and TBC.

clarity. For the expansion case, the analytical stress field is outstandingly precise and the stress distribution completely resembles the computational and average computational stresses. However, for the shear case various conclusions can be drawn. For incl./matr. $=0.1$, the average stresses due to DBC overestimate the analytical stresses both in the matrix and particle. On the other hand, the average stresses due to TBC underestimate the analytical stresses both in the matrix and particle. When incl. $/$ matr. $=1$, for size $=0.01$, the computational average stresses due to DBC and TBC overestimate and underestimate the analytical stresses in both phases, respectively. When size $=100$, the same result holds for the average stress in the matrix. However, the average stress in the fiber due to TBC overestimate the analytical stress with
DBC underestimating it. Finally, for incl./matr. $=10$, the computational average stresses similarly overestimate and underestimate the analytical stresses when size $=0.01$. DBC results in the least average stress in the matrix where as TBC yields the highest value when size $=100$.

\section{Discussion and comparison with literature}

In this section, we carry out a comprehensive comparison between our results and the available analytical estimates in the literature for the elastic and cohesive interfaces. In doing so, the overall moduli of particulate composites are examined for various stiffness ratios at different sizes. As mentioned earlier, suitable choices of the general interface 


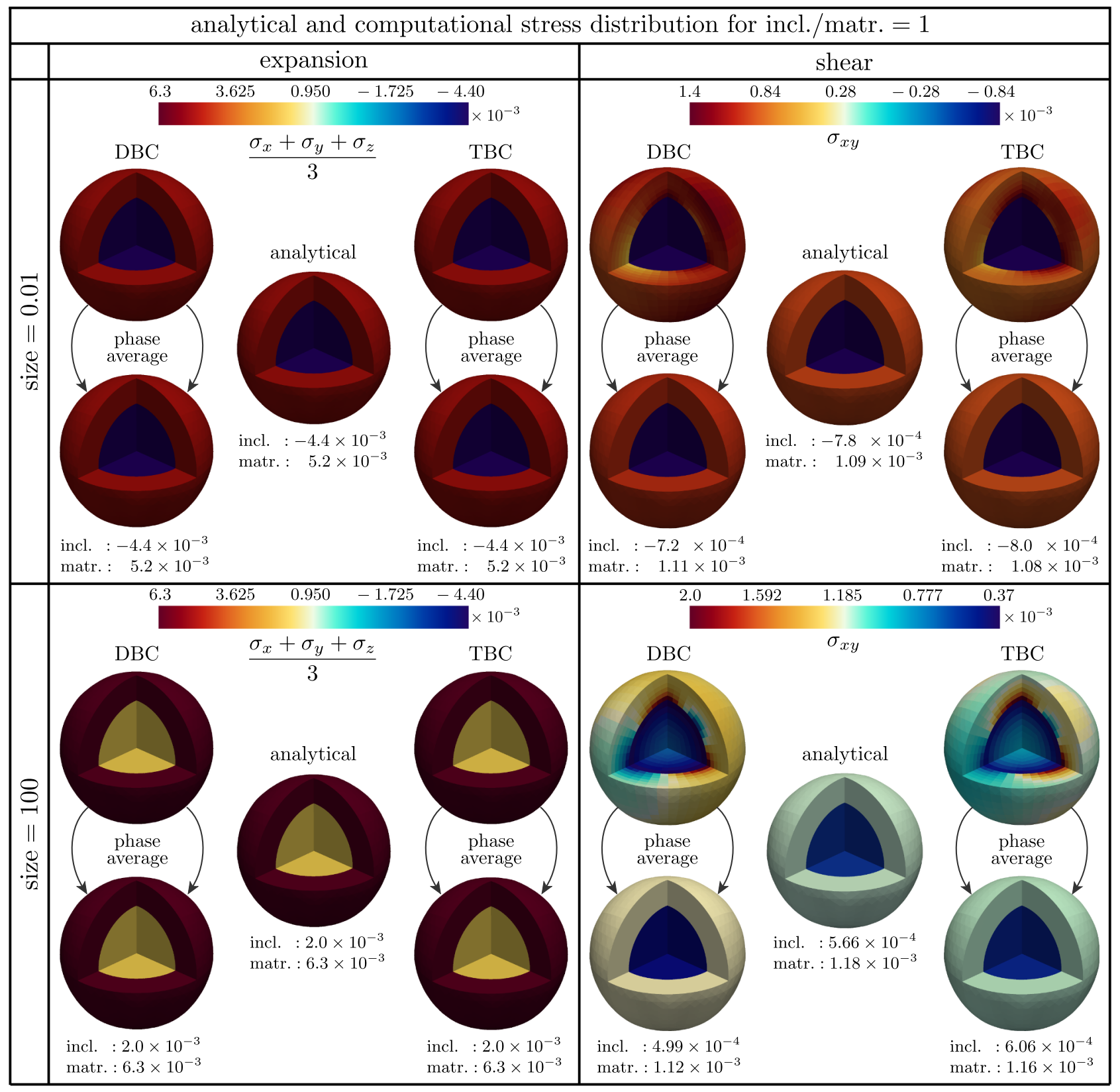

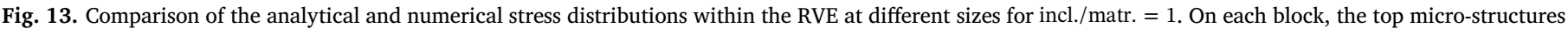

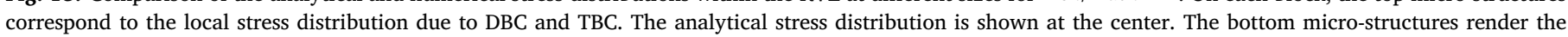
average of the computational stresses due to DBC and TBC.

parameters can recover any of elastic, cohesive or perfect interfaces, see Fig. 1 for more clarity. The elastic (stress-type) interface model here is recovered by setting $\bar{k} \rightarrow \infty$ in our general interface model. On the contrary, the cohesive (spring-type) interface model is recovered by setting $\bar{\lambda}=0$ and $\bar{\mu}=0$. Our explicit expressions for the bulk modulus in these limit cases reduce to

$$
\mathrm{M}_{\kappa}=\frac{r_{1}\left[3 \kappa_{1}\left[3 \kappa_{2}+4 f \mu_{2}\right]+12 \kappa_{2} \mu_{2}[1-f]\right]+4[\bar{\lambda}+\bar{\mu}]\left[3 \kappa_{2}+4 f \mu_{2}\right]}{3 r_{1}\left[3 \kappa_{1}+4 \mu_{2}+3 f\left[\kappa_{2}-\kappa_{1}\right]\right]+12[\bar{\lambda}+\bar{\mu}][1-f]} .
$$

$\mathrm{M}_{\mathcal{K}}=\frac{\bar{k} r_{1}\left[3 \kappa_{1}\left[3 \kappa_{2}+4 f \mu_{2}\right]+12 \kappa_{2} \mu_{2}[1-f]\right]+36 \kappa_{1} \kappa_{2} \mu_{2}[1-f]}{3 \bar{k} r_{1}\left[3 \kappa_{1}+4 \mu_{2}+3 f\left[\kappa_{2}-\kappa_{1}\right]\right]+9 \kappa_{1}\left[4 \mu_{2}+3 f \kappa_{2}\right]}$.
For the shear modulus, the final expressions for the bounds and estimate (Eqs. (28), (33) and (39)) remain the same, however, special treatments are required while assembling the corresponding matrices in Eqs. (A.1), (A.3) and (A.6). For the sake of brevity, we omit the associated derivations for shear modulus as it does not render a short closed-form explicit solution. Fig. 15 shows the effective moduli versus size for various stiffness ratios for the elastic interface parameters of $\bar{\lambda}=\bar{\mu}=1$. In this example, we compare our results with four other analytical estimates developed in (Duan et al., 2007; 2005; Gu et al., 2014; Zemlyanova and Mogilevskaya, 2018). Note, the framework developed by Zemlyanova and Mogilevskaya (2018) accounts for the Steigmann-Ogden model, but we simplified it to the Gurtin-Murdoch interface model suitable for the comparison, see also (Kushch et al., 


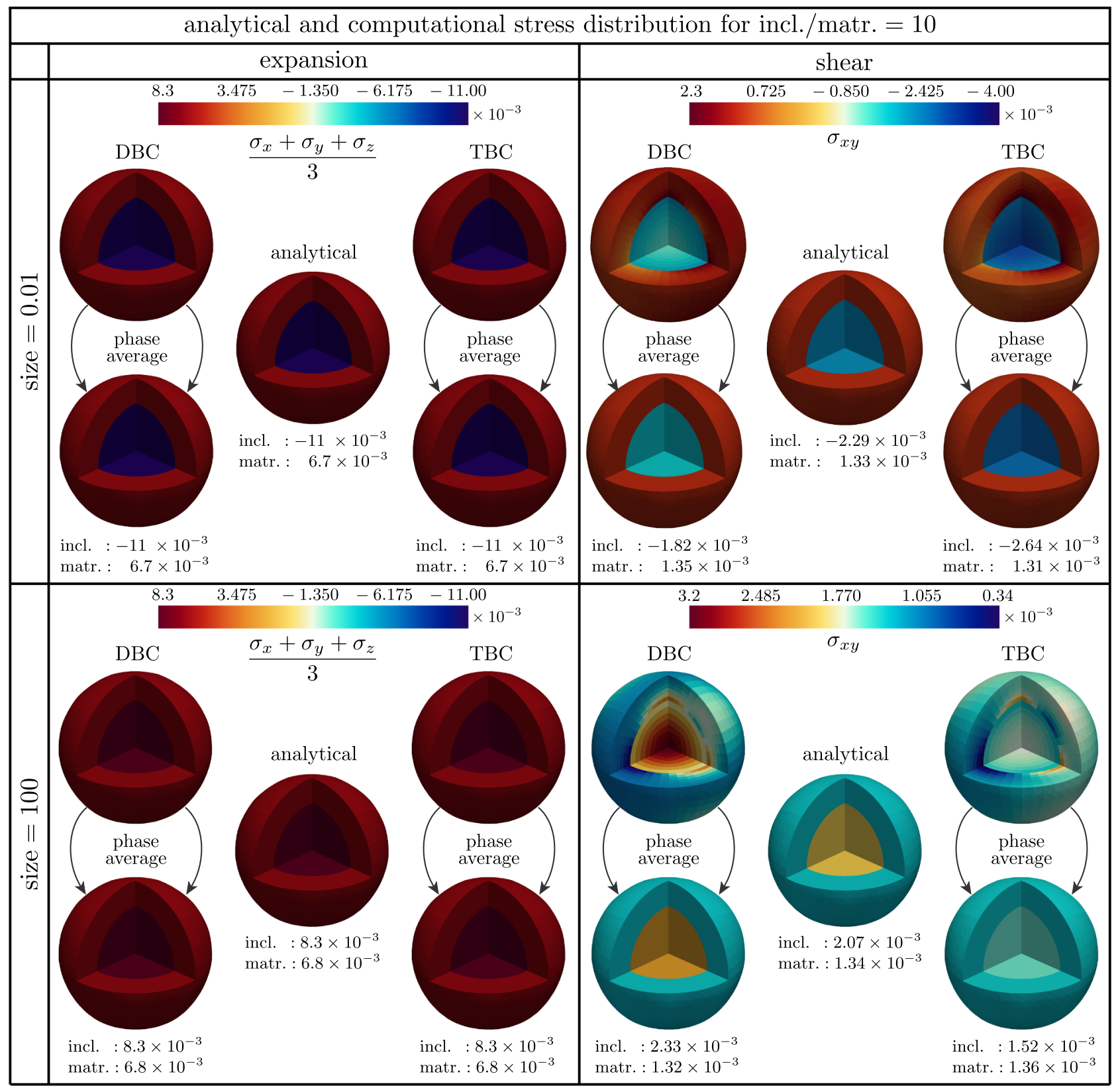

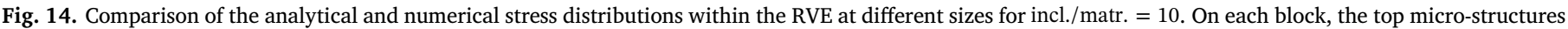

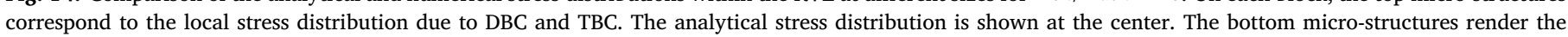
average of the computational stresses due to DBC and TBC.

2013). We observe an excellent agreement between all the solutions for the bulk modulus. For the case of shear modulus, a remarkable agreement is observed between our generalized self-consistent method and the solution proposed by Duan et al. (2007) and Gu et al. (2014). Our Mori-Tanaka method also coincides with the solution developed by Duan et al. (2005). Moreover, the solution developed by Zemlyanova and Mogilevskaya (2018) coincides with the lower bound at small sizes and tends to converge to the Mori-Tanaka method as the size increases. It is noteworthy that the closed-form expression for the bulk modulus proposed by Nazarenko et al. (2017) is also in perfect accordance with these results. Fig. 16 shows the effective moduli versus size for various stiffness ratios for the cohesive interface parameter of $\bar{k}=1$. In this example, we compare our results with two other analytical estimates developed by Duan et al. (2007) and Gu et al. (2014). Similar to the previous case, all the results coincide for the case of bulk modulus. For the shear modulus, there is a remarkable agreement our generalized self-consistent method and the solution proposed by Duan et al. (2007) and Gu et al. (2014). It shall be noted that Duan et al. (2005) obtain the effective properties using three different types of micromechanics schemes, i.e. the Mori-Tanaka method (MTM), the composite spheres assemblage (CSA) and the generalized self-consistent method (GSCM). On the other hand, Gu et al. (2014), Duan et al. (2007) and Zemlyanova and Mogilevskaya (2018) use a two-step procedure as follows. Firstly, they 


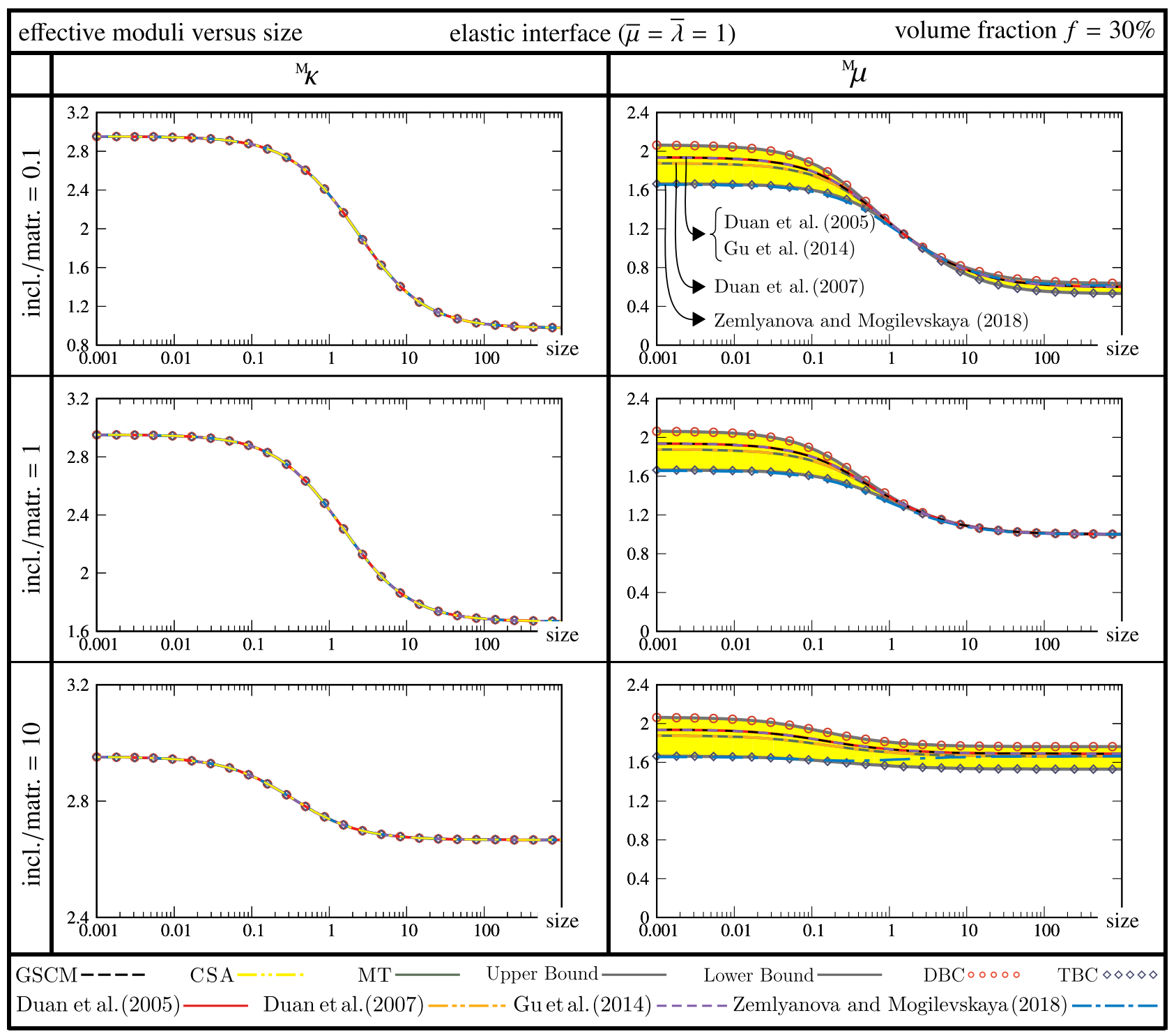

Fig. 15. Comparison of the overall moduli for the elastic interface with the available closed-form expressions in the literature.

identify an equivalent particle that substitutes the particle-plus-interface and secondly, they utilize standard homogenization schemes. In the second step though, Gu et al. (2014) and Duan et al. (2007) consider the GSCM, while Zemlyanova and Mogilevskaya (2018) utilize the Maxwell's homogenization approach. For further details about Maxwell's homogenization approach and its utility for a broad range of applications, see Sevostianov et al. (2019). It is well-established that the effective bulk modulus of a particle reinforced composite obtained with Maxwell's scheme is identical to that obtained with all the other classical homogenization schemes. This property explains the excellent agreement between the results from all various homogenization techniques on the left column of Fig. 15. On the other hand, Maxwell-based estimates for the effective shear modulus are different from those obtained with generalized self-consistent type schemes. This feature explains the difference between the results on the right column of Fig. 15. In the classical case, the Maxwell-based estimates coincide with the expressions for the lower Hashin-Shtrikman bound if the particles are stiffer than the matrix. On the contrary, if the matrix is stiffer than the particles, the Maxwell-based estimates coincide with the expressions for the upper Hashin-Shtrikman bound. This property seems to remain valid even in the presence of generalized interfaces between the matrix and particles. Note that a key feature of this contribution is to verify the analytical estimates with computational simulations using the finite element method, for the first time.

\section{Conclusion}

We established two novel techniques to determine the overall behavior of particulate composites via extending homogenization to account for general interfaces hence, size effects. An interface-enhanced composite cylinder assemblage (CSA) approach and generalized selfconsistent method (GSCM) were developed to incorporate interfaces resulting in estimates and bounds for the effective properties of composites. Explicit formulations for the overall bulk modulus and semiexplicit formulations for the overall shear modulus of particulate composites accounting for general interfaces were presented for the 


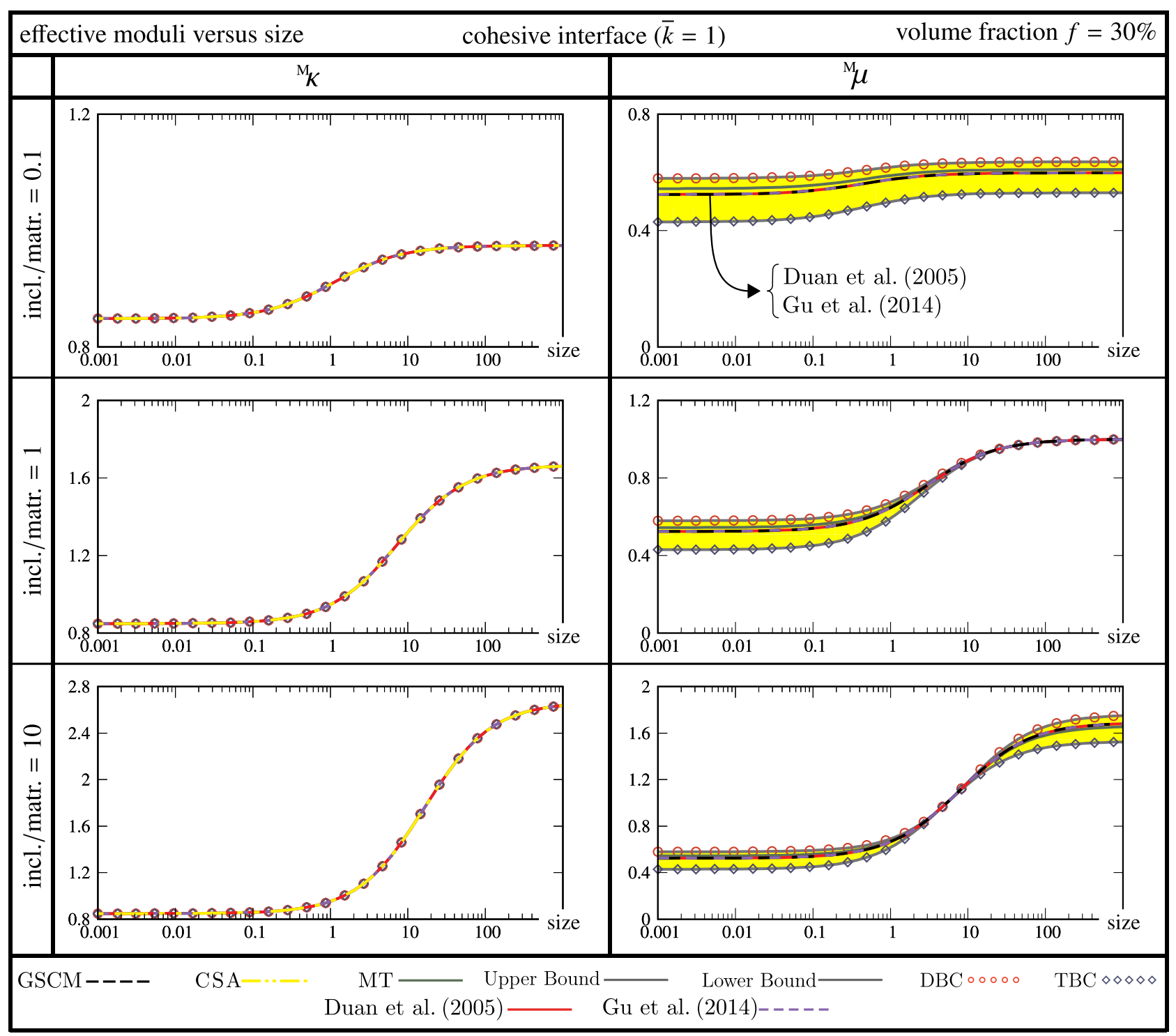

Fig. 16. Comparison of the overall moduli for the cohesive interface with the available closed-form expressions in the literature.

first time. Afterwards, we developed a generalized interface enhanced Mori-Tanaka to study size effects in particulate composites, verified by numerical computations. This approach not only provides estimates for the overall properties, but also identifies concentration tensors in all phases of the composite. As a result, the state of stress and strain in the matrix, particle and the interface can be determined which provides a significant insight into the computational design of composites accounting for generalized interfaces and size effects. A perfect agreement is observed between the proposed analytical estimates and the computational results obtained using the finite element method. Our proposed methodology is versatile and can recover any of elastic, cohesive or perfect interface models. All of the observations demonstrate that the overall material response in the presence of the general interface is, in principle, complicated. We believe that this manuscript deepens our understanding of the interface effects and size-dependent behavior of composites which paves the way towards computational metamaterial design.

\section{Appendix A. System of equations for the estimate and bounds on the shear modulus}

In this section we elaborate on the system of equations used to obtain the estimate and the bounds on the macroscopic shear modulus explained in Section 3.2.

\section{A1. Strain bound on the shear modulus}

The displacement fields in the matrix and the particle were given in Eq. (24) which has eight unknowns of $\Xi_{1}^{(1)}, \Xi_{2}^{(1)}, \Xi_{3}^{(1)}, \Xi_{4}^{(1)}, \Xi_{1}^{(2)}, \Xi_{2}^{(2)}, \Xi_{3}^{(2)}$ and $\Xi_{4}^{(2)}$. We concluded that since the displacement fields at the center of the RVE must be finite, $\Xi_{3}^{(1)}$ and $\Xi_{4}^{(1)}$ must vanish. For the remaining six unknowns we apply the boundary and interface conditions (25) resulting in the below system of equations 


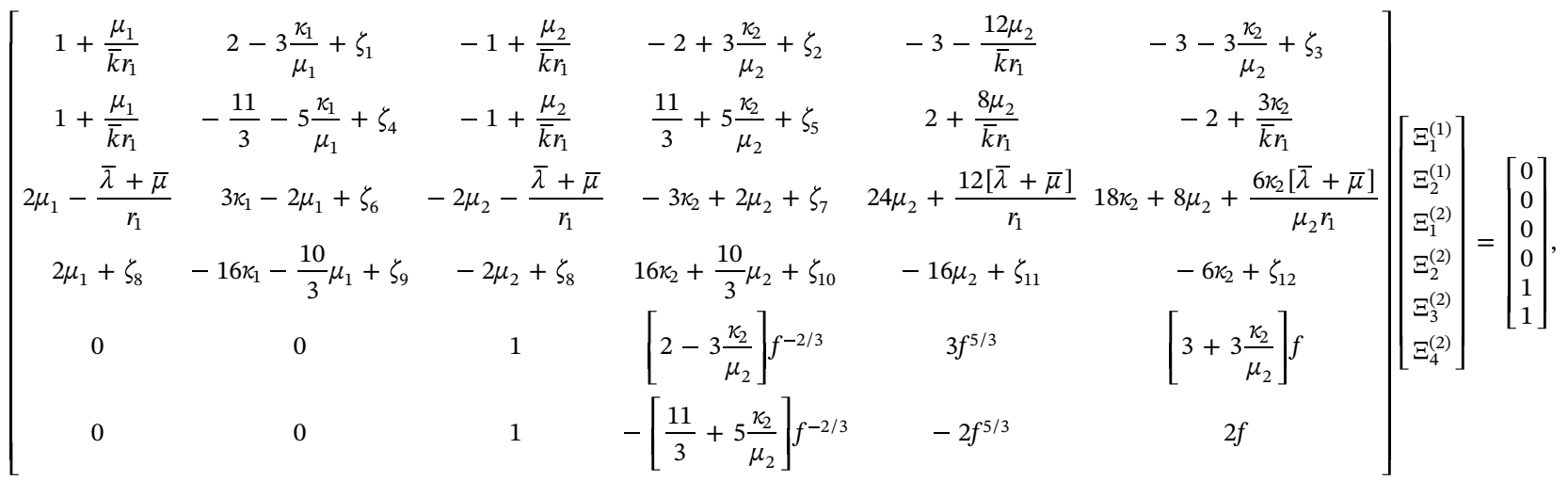

with

$\zeta_{1}=\frac{3 \kappa_{1}-2 \mu_{1}}{2 \bar{k} r_{1}}, \quad \zeta_{5}=-\frac{24 \kappa_{2}+5 \mu_{2}}{3 \bar{k} r_{1}}, \quad \zeta_{9}=-\frac{\kappa_{1}[9 \bar{\lambda}+19 \bar{\mu}]}{\mu_{1} r_{1}}-\frac{[45 \bar{\lambda}+67 \bar{\mu}]}{3 r_{1}}$,

$\zeta_{2}=\frac{3 \kappa_{2}-2 \mu_{2}}{2 \bar{k} r_{1}}, \quad \zeta_{6}=\frac{\left[9 \kappa_{1}+15 \mu_{1}\right][\bar{\lambda}+\bar{\mu}]}{\mu_{1} r_{1}}, \quad \zeta_{10}=-\frac{\kappa_{2}[9 \bar{\lambda}+19 \bar{\mu}]}{\mu_{2} r_{1}}-\frac{[45 \bar{\lambda}+67 \bar{\mu}]}{3 r_{1}}$,

$\zeta_{3}=-\frac{9 \kappa_{2}+4 \mu_{2}}{\bar{k} r_{1}}, \quad \zeta_{7}=\frac{\left[9 \kappa_{2}+15 \mu_{2}\right][\bar{\lambda}+\bar{\mu}]}{\mu_{2} r_{1}}, \quad \zeta_{11}=-\frac{4[3 \bar{\lambda}+4 \bar{\mu}]}{r_{1}}$,

$\zeta_{4}=-\frac{24 \kappa_{1}+5 \mu_{1}}{3 \bar{k} r_{1}}, \quad \zeta_{8}=\frac{[\bar{\lambda}+\bar{\mu}]+2 \bar{\mu}}{r_{1}}, \quad \zeta_{12}=\frac{-6 \kappa_{2}[\bar{\lambda}+\bar{\mu}]+4 \mu_{2} \bar{\mu}}{\mu_{2} r_{1}}$.

\section{A2. Stress bound on shear modulus}

The far field stress field in this case led to the displacement field according to Eq. (24) with the eight unknowns $\Xi_{1}^{(1)}, \Xi_{2}^{(1)}, \Xi_{3}^{(1)}, \Xi_{4}^{(1)}, \Xi_{1}^{(2)}, \Xi_{2}^{(2)}, \Xi_{3}^{(2)}$ and $\Xi_{4}^{(2)}$. We concluded that since the displacement fields at the center of the RVE must be finite, $\Xi_{3}^{(1)}$ and $\Xi_{4}^{(1)}$ must vanish. For the remaining six unknowns we apply the boundary and interface conditions (30) resulting in the below system of equations

\begin{tabular}{|c|c|c|c|c|c|c|c|}
\hline $1+\frac{\mu_{1}}{\bar{k} r_{1}}$ & $2-3 \frac{\kappa_{1}}{\mu_{1}}+\zeta_{1}$ & $-1+\frac{\mu_{2}}{\bar{k} r_{1}}$ & $-2+3 \frac{\kappa_{2}}{\mu_{2}}+\zeta_{2}$ & $-3-\frac{12 \mu_{2}}{\bar{k} r_{1}}$ & $-3-3 \frac{\kappa_{2}}{\mu_{2}}+\zeta_{3}$ & & \\
\hline $1+\frac{\mu_{1}}{\bar{k} r_{1}}$ & $-\frac{11}{3}-5 \frac{\kappa_{1}}{\mu_{1}}+\zeta_{4}$ & $-1+\frac{\mu_{2}}{\bar{k} r_{1}}$ & $\frac{11}{3}+5 \frac{\kappa_{2}}{\mu_{2}}+\zeta_{5}$ & $2+\frac{8 \mu_{2}}{\bar{k} r_{1}}$ & $-2+\frac{3 \kappa_{2}}{\bar{k} r_{1}}$ & $\left.\begin{array}{l}\Xi_{1}^{(1)} \\
\Xi_{2}^{(1)}\end{array}\right]$ & {$[0]$} \\
\hline $2 \mu_{1}-\frac{\bar{\lambda}+\bar{\mu}}{r_{1}}$ & $3 \kappa_{1}-2 \mu_{1}+\zeta_{6}$ & $-2 \mu_{2}-\frac{\bar{\lambda}+\bar{\mu}}{r_{1}}$ & $-3 \kappa_{2}+2 \mu_{2}+\zeta_{7}$ & $24 \mu_{2}+\frac{12[\bar{\lambda}+\bar{\mu}]}{r_{1}}$ & $18 \kappa_{2}+8 \mu_{2}+\frac{6 \kappa_{2}[\bar{\lambda}+\bar{\mu}]}{\mu_{2} r_{1}}$ & $\begin{array}{l}\Xi_{1}^{(2)} \\
\Xi_{2}^{(2)}\end{array}$ & $=\mid \begin{array}{l}0 \\
0 \\
0\end{array}$ \\
\hline $2 \mu_{1}+\zeta_{8}$ & $-16 \kappa_{1}-\frac{10}{3} \mu_{1}+\zeta_{9}$ & $-2 \mu_{2}+\zeta_{8}$ & $16 \kappa_{2}+\frac{10}{3} \mu_{2}+\zeta_{10}$ & $-16 \mu_{2}+\zeta_{11}$ & $-6 \kappa_{2}+\zeta_{12}$ & $\begin{array}{l}\Xi_{2} \\
\Xi_{3}^{(2)}\end{array}$ & {$\left[\begin{array}{l}1 \\
1\end{array}\right]$} \\
\hline 0 & 0 & $2 \mu_{2}$ & {$\left[3 \kappa_{2}-2 \mu_{2}\right] f^{-2 / 3}$} & $-24 \mu_{2} f^{5 / 3}$ & $-\left[18 \kappa_{2}+8 \mu_{2}\right] f$ & $\Xi_{4}^{(2)}$ & \\
\hline 0 & 0 & $2 \mu_{2}$ & $-\left[16 \kappa_{2}+\frac{10}{3} \mu_{2}\right] f^{-2 / 3}$ & $16 \mu_{2} f^{5 / 3}$ & $6 \kappa_{2} f$ & & \\
\hline
\end{tabular}

with

$\zeta_{1}=\frac{3 \kappa_{1}-2 \mu_{1}}{2 \bar{k} r_{1}}, \quad \zeta_{5}=-\frac{24 \kappa_{2}+5 \mu_{2}}{3 \bar{k} r_{1}}, \quad \zeta_{9}=-\frac{\kappa_{1}[9 \bar{\lambda}+19 \bar{\mu}]}{\mu_{1} r_{1}}-\frac{[45 \bar{\lambda}+67 \bar{\mu}]}{3 r_{1}}$,

$\zeta_{2}=\frac{3 \kappa_{2}-2 \mu_{2}}{2 \bar{k} r_{1}}, \quad \zeta_{6}=\frac{\left[9 \kappa_{1}+15 \mu_{1}\right][\bar{\lambda}+\bar{\mu}]}{\mu_{1} r_{1}}, \quad \zeta_{10}=-\frac{\kappa_{2}[9 \bar{\lambda}+19 \bar{\mu}]}{\mu_{2} r_{1}}-\frac{[45 \bar{\lambda}+67 \bar{\mu}]}{3 r_{1}}$,

$\zeta_{3}=-\frac{9 \kappa_{2}+4 \mu_{2}}{\bar{k} r_{1}}, \quad \zeta_{7}=\frac{\left[9 \kappa_{2}+15 \mu_{2}\right][\bar{\lambda}+\bar{\mu}]}{\mu_{2} r_{1}}, \quad \zeta_{11}=-\frac{4[3 \bar{\lambda}+4 \bar{\mu}]}{r_{1}}$

$\zeta_{4}=-\frac{24 \kappa_{1}+5 \mu_{1}}{3 \bar{k} r_{1}}, \quad \zeta_{8}=\frac{[\bar{\lambda}+\bar{\mu}]+2 \bar{\mu}}{r_{1}}, \quad \zeta_{12}=\frac{-6 \kappa_{2}[\bar{\lambda}+\bar{\mu}]+4 \mu_{2} \bar{\mu}}{\mu_{2} r_{1}}$.

\section{A3. Effective shear modulus}

For this problem the displacement fields in the particle and the matrix are given in Eq. (24) and the displacement field in the effective medium is given in Eq. (35). This problem contains ten unknown of $\Xi_{1}^{(1)}, \Xi_{2}^{(1)}, \Xi_{3}^{(1)}, \Xi_{4}^{(1)}, \Xi_{1}^{(2)}, \Xi_{2}^{(2)}, \Xi_{3}^{(2)}, \Xi_{4}^{(2)}, \Xi_{3}^{\text {(eff) }}$ and $\Xi_{4}^{\text {(eff) }}$. We concluded that since at the center of the RVE the displacement field needs to be finite, $\Xi_{3}^{(1)}$ and $\Xi_{4}^{(1)}$ must be zero. Substitution of the stress and displacement fields in Eq. (38) yielded $\Xi_{4}^{\text {(eff) }}=0$. For the remaining unknowns we apply the boundary and interface conditions (37) resulting in the below system of equations 
$Q\left[\begin{array}{l}\Xi_{1}^{(1)} \\ \Xi_{2}^{(1)} \\ \Xi_{1}^{(2)} \\ \Xi_{2}^{(2)} \\ \Xi_{3}^{(2)} \\ \Xi_{4}^{(2)}\end{array}\right]=\left[\begin{array}{l}0 \\ 0 \\ 0 \\ 0 \\ 1 \\ 1\end{array}\right]+\left[\begin{array}{c}0 \\ 0 \\ 0 \\ 0 \\ 3 \\ -2\end{array}\right] f^{5 / 3} \Xi_{3}^{\text {eff }}$

where

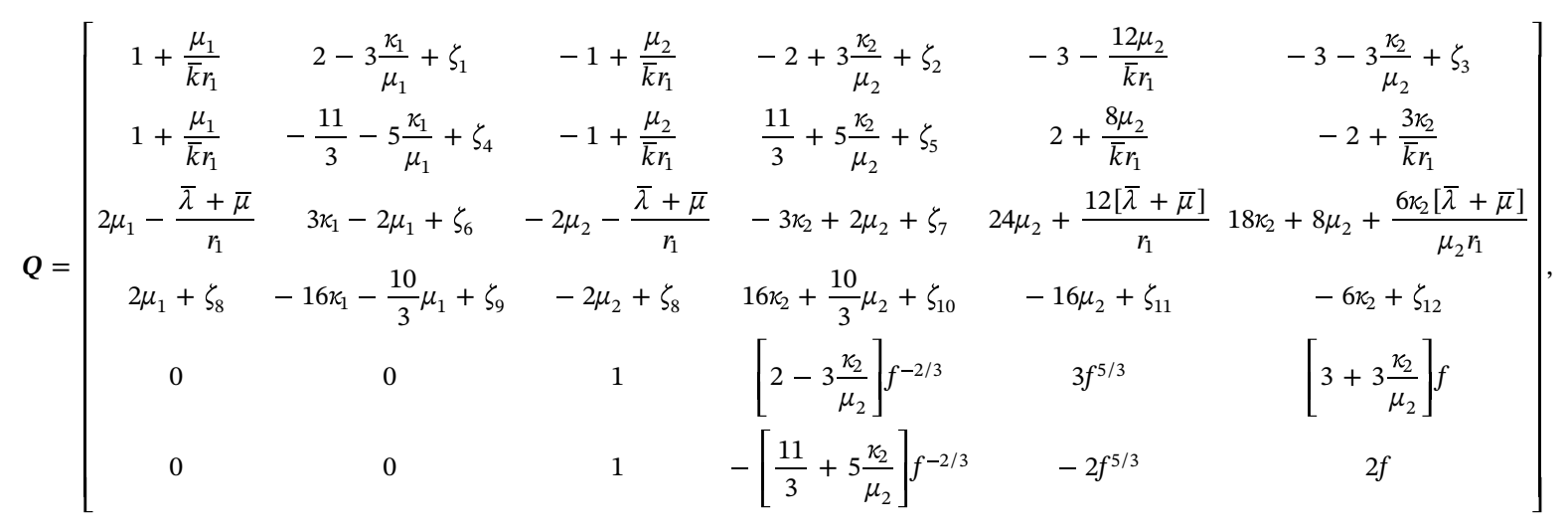

with

$\zeta_{1}=\frac{3 \kappa_{1}-2 \mu_{1}}{2 \bar{k} r_{1}}, \quad \zeta_{5}=-\frac{24 \kappa_{2}+5 \mu_{2}}{3 \bar{k} r_{1}}, \quad \zeta_{9}=-\frac{\kappa_{1}[9 \bar{\lambda}+19 \bar{\mu}]}{\mu_{1} r_{1}}-\frac{[45 \bar{\lambda}+67 \bar{\mu}]}{3 r_{1}}$,

$\zeta_{2}=\frac{3 \kappa_{2}-2 \mu_{2}}{2 \bar{k} r_{1}}, \quad \zeta_{6}=\frac{\left[9 \kappa_{1}+15 \mu_{1}\right][\bar{\lambda}+\bar{\mu}]}{\mu_{1} r_{1}}, \quad \zeta_{10}=-\frac{\kappa_{2}[9 \bar{\lambda}+19 \bar{\mu}]}{\mu_{2} r_{1}}-\frac{[45 \bar{\lambda}+67 \bar{\mu}]}{3 r_{1}}$,

$\zeta_{3}=-\frac{9 \kappa_{2}+4 \mu_{2}}{\bar{k} r_{1}}, \quad \zeta_{7}=\frac{\left[9 \kappa_{2}+15 \mu_{2}\right][\bar{\lambda}+\bar{\mu}]}{\mu_{2} r_{1}}, \quad \zeta_{11}=-\frac{4[3 \bar{\lambda}+4 \bar{\mu}]}{r_{1}}$,

$\zeta_{4}=-\frac{24 \kappa_{1}+5 \mu_{1}}{3 \bar{k} r_{1}}, \quad \zeta_{8}=\frac{[\bar{\lambda}+\bar{\mu}]+2 \bar{\mu}}{r_{1}}, \quad \zeta_{12}=\frac{-6 \kappa_{2}[\bar{\lambda}+\bar{\mu}]+4 \mu_{2} \bar{\mu}}{\mu_{2} r_{1}}$.

The obtained system of equations though is nonlinear hence, requiring a special treatment. We express the solution of the above system in the form $\left[\begin{array}{l}\Xi_{1}^{(1)} \\ \Xi_{2}^{(1)} \\ \Xi_{1}^{(2)} \\ \Xi_{2}^{(2)} \\ \Xi_{3}^{(2)} \\ \Xi_{4}^{(2)}\end{array}\right]=\left[\begin{array}{l}g_{1} \\ g_{2} \\ a_{1} \\ a_{2} \\ a_{3} \\ a_{4}\end{array}\right]+\left[\begin{array}{l}h_{1} \\ h_{2} \\ b_{1} \\ b_{2} \\ b_{3} \\ b_{4}\end{array}\right] f^{5 / 3} \Xi_{3}^{\text {(eff) }}$

Using (A.8), the last two conditions of Eq. (36) are written as

$a_{5}+b_{5} f^{5 / 3} \Xi_{3}^{(\text {eff })}=2^{\mathrm{M}} \mu-24{ }^{\mathrm{M}} \mu f^{5 / 3} \Xi_{3}^{\text {(eff) }}, \quad a_{6}+b_{6} f^{5 / 3} \Xi_{3}^{\text {(eff) }}=2^{\mathrm{M} \mu}+16^{\mathrm{M}} \mu f^{5 / 3} \Xi_{3}^{\text {(eff) }}$,

with

$a_{5}=2 \mu_{2} a_{1}+\left[3 \kappa_{2}-2 \mu_{2}\right] f^{-2 / 3} a_{2}-24 \mu_{2} f^{5 / 3} a_{3}-\left[18 \kappa_{2}+8 \mu_{2}\right] f a_{4}$,

$a_{6}=2 \mu_{2} a_{1}-\left[16 \kappa_{2}+\frac{10}{3} \mu_{2}\right] f^{-2 / 3} a_{2}+16 \mu_{2} f^{5 / 3} a_{3}+6 \kappa_{2} f a_{4}$,

$b_{5}=2 \mu_{2} b_{1}+\left[3 \kappa_{2}-2 \mu_{2}\right] f^{-2 / 3} b_{2}-24 \mu_{2} f^{5 / 3} b_{3}-\left[18 \kappa_{2}+8 \mu_{2}\right] f b_{4}$,

$b_{6}=2 \mu_{2} b_{1}-\left[16 \kappa_{2}+\frac{10}{3} \mu_{2}\right] f^{-2 / 3} b_{2}+16 \mu_{2} f^{5 / 3} b_{3}+6 \kappa_{2} f b_{4}$.

Subtracting (A.9) $)_{1}$ from (A.9) 2 gives

$f^{5 / 3} \Xi_{3}^{(\text {eff })}=\frac{a_{6}-a_{5}}{40^{\mathrm{M}} \mu+b_{5}-b_{6}}$.

Substituting the final result in (A.9) ${ }_{1}$, after some algebra we obtain the below quadratic equation

$80^{\mathrm{M}} \mu^{2}-2\left[b_{6}-b_{5}+12 a_{6}+8 a_{5}\right]^{\mathrm{M}} \mu+a_{5} b_{6}-b_{5} a_{6}=0$.

From the two possible solutions the positive value is the macroscopic shear modulus. 


\section{References}

Alfano, G., Crisfield, M.A., 2001. Finite element interface models for the delamination analysis of laminated composites: mechanical and computational issues. Int. J. Numer. Methods Eng. 50, 1701-1736.

Altenbach, H., Eremeyev, V.A., 2011. On the shell theory on the nanoscale with surface stresses. Int. J. Eng. Sci. 49, 1294-1301.

Barenblatt, G.I., 1959. The formation of equilibrium cracks during brittle fracture. general ideas and hypotheses. Axially-symmetric cracks. J. Appl. Math. Mech. 23, 622-636.

Barenblatt, G.I., 1962. The mathematical theory of equilibrium of crack in brittle fracture. Adv. Appl. Mech. 7, 55-129.

Benveniste, Y., 2006. A general interface model for a three-dimensional curved thin anisotropic interphase between two anisotropic media. J. Mech. Phys. Solids 54, $708-734$

Benveniste, Y., Dvorak, G.J., Chen, T., 1989. Stress fields in composites with coated inclusions. Mech. Mater. 7, 305-317.

Benveniste, Y., Miloh, T., 2001. Imperfect soft and stiff interfaces in two-dimensional elasticity. Mech. Mater. 33, 309-323.

Brassart, L., Inglis, H.M., Delannay, L., Doghri, I., Geubelle, P.H., et al., 2009. An extended Mori-Tanaka homogenization scheme for finite strain modeling of debonding in particle-reinforced elastomers. Comput. Mater. Sci 45, 611-616.

Brisard, S., Dormieux, L., Kondo, D., 2010. Hashin-Shtrikman bounds on the shear modulus of a nanocomposite with spherical inclusions and interface effects. Comput. Mater. Sci 50, 403-410.

Charalambakis, N., Chatzigeorgiou, G., Chemisky, Y., Meraghni, F., et al., 2018. Mathematical homogenization of inelastic dissipative materials: a survey and recent progress. Continuum Mech. Thermodyn. 30, 1-51.

Chatzigeorgiou, G., Javili, A., Steinmann, P., 2015. Multiscale modelling for composites with energetic interfaces at the micro- or nanoscale. Math. Mech. Solids 20, $1130-1145$.

Chatzigeorgiou, G., Meraghni, F., Javili, A., 2017. Generalized interfacial energy and size effects in composites. J. Mech. Phys. Solids 106, 257-282.

Chen, T., Chiu, M.S., Weng, C.N., 2006. Derivation of the generalized Young-Laplace equation of curved interfaces in nanoscaled solids. J. Appl. Phys. 100, 074308

Chhapadia, P., Mohammadi, P., Sharma, P., 2011. Curvature-dependent surface energy and implications for nanostructures. J. Mech. Phys. Solids 59, 2103-2115.

Christensen, R.M., Lo, K.H., 1979. Solutions for effective shear properties in three phase sphere and cylinder models. J. Mech. Phys. Solids 27, 315-330.

Cordero, N.M., Forest, S., Busso, E.P., 2016. Second strain gradient elasticity of nanoobjects. J. Mech. Phys. Solids 97, 92-124.

Daher, N., Maugin, G.A., 1986. The method of virtual power in continuum mechanics application to media presenting singular surfaces and interfaces. Acta Mech. 60, 217-240.

Davydov, D., Javili, A., Steinmann, P., 2013. On molecular statics and surface-enhanced continuum modeling of nano-structures. Comput. Mater. Sci 69, 510-519.

dell'Isola, F., Romano, A., 1987. On the derivation of thermomechanical balance equations for continuous system with a nonmaterial interface. Int. J. Eng. Sci. 25, 1459-1468.

Despringre, N., Chemisky, Y., Bonnay, K., Meraghni, F., et al., 2016. Micromechanical modeling of damage and load transfer in particulate composites with partially debonded interface. Compos. Struct. 155, 77-88.

Dimitri, R., Trullo, M., De Lorenzis, L., Zavarise, G., et al., 2015. Coupled cohesive zone models for mixed-mode fracture: a comparative study. Eng. Fract. Mech. 148, 145-179.

Dingreville, R., Hallil, A., Berbenni, S., 2014. From coherent to incoherent mismatched interfaces: a generalized continuum formulation of surface stresses. J. Mech. Phys. Solids 72, 40-60.

Dingreville, R., Qu, J., 2008. Interfacial excess energy, excess stress and excess strain in elastic solids: planar interfaces. J. Mech. Phys. Solids 56, 1944-1954.

Dinzart, F., Sabar, H., 2017. New micromechanical modeling of the elastic behavior of composite materials with ellipsoidal reinforcements and imperfect interfaces. Int. J. Solids Struct. 108, 254-262.

Duan, H.L., Karihaloo, B.L., 2007. Effective thermal conductivities of heterogeneous media containing multiple imperfectly bonded inclusions. Phys. Rev. B 75, 064206.

Duan, H.L., Wang, J., Huang, Z.P., Karihaloo, B.L., et al., 2005. Size-dependent effective elastic constants of solids containing nano-inhomogeneities with interface stress. J. Mech. Phys. Solids 53, 1574-1596.

Duan, H.L., Wang, J., Karihaloo, B.L., 2009. Theory of elasticity at the nanoscale. Adv. Appl. Mech. 42, 1-68.

Duan, H.L., Yi, X., Huang, Z.P., Wang, J., et al., 2007. A unified scheme for prediction of effective moduli of multiphase composites with interface effects. Part i: theoretical framework. Mech. Mater. 39, 81-93.

Dugdale, D.S., 1960. Yielding of steel sheets containing slits. J. Mech. Phys. Solids 8, 100-104.

Elsner, B.A.M., Müller, S., Bargmann, S., Weissmüller, J., et al., 2017. Surface excess elasticity of gold: ab initio coefficients and impact on the effective elastic response of nanowires. Acta Mater. 124, 468-477.

Entchev, P.B., Lagoudas, D.C., 2002. Modeling porous shape memory alloys using micromechanical averaging techniques. Mech. Mater. 34, 1-24.

Fagerström, M., Larsson, R., 2006. Theory and numerics for finite deformation fracture modelling using strong discontinuities. Int. J. Numer. Methods Eng. 66, 911-948.

Fedotov, A., 2018. Interface model of homogenization for analysing the influence of in clusion size on the elastic properties of composites. Compos. Part B 152, 241-247.

Firooz, S., Chatzigeorgiou, G., Meraghni, F., Javili, A., et al., 2019. Bounds on size effects in composites via homogenization accounting for general interfaces. Continuum Mech. Thermodyn. https://doi.org/10.1007/s00161-019-00796-w.

Firooz, S., Javili, A., 2019. Understanding the role of general interfaces in the overall behavior of composites and size effects. Comput. Mater. Sci 162, 245-254.

Firooz, S., Saeb, S., Chatzigeorgiou, G., Meraghni, F., Steinmann, P., Javili, A., et al., 2019. Systematic study of homogenization and the utility of circular simplified representative volume element. Math. Mech. Solids 24, 2961-2985.

Fried, E., Gurtin, M.E., 2007. Thermomechanics of the interface between a body and its environment. Continuum Mech. Thermodyn. 19, 253-271.

Fried, E., Todres, R.E., 2005. Mind the gap: the shape of the free surface of a rubber-like material in proximity to a rigid contactor. J. Elast. 80, 97-151.

Fritzen, F., Leuschner, M., 2015. Nonlinear reduced order homogenization of materials including cohesive interfaces. Comput. Mech. 56, 131-151.

Gasser, T.C., Holzapfel, G.A., 2003. Geometrically non-linear and consistently linearized embedded strong discontinuity models for 3D problems with an application to the dissection analysis of soft biological tissues. Comput. Methods Appl. Mech. Eng. 192, 5059-5098.

Gitman, I.M., Askes, H., Aifantis, E.C., 2005. The representative volume size in static and dynamic micro-macro transitions. Int. J. Fract. 135, 3-9.

Goudarzi, M., Simone, A., 2019. Fiber neutrality in fiber-reinforced composites: evidence from a computational study. Int. J. Solids Struct. 156-157, 14-28.

Gu, S.T., Liu, J.T., He, Q.C., 2014. Size-dependent effective elastic moduli of particulate composites with interfacial displacement and traction discontinuities. Int. J. Solids Struct. 51, 2283-2296.

Gu, S.T., Monteiro, E., He, Q.C., 2011. Coordinate-free derivation and weak formulation of a general imperfect interface model for thermal conduction in composites. Compos. Sci. Technol. 71, 1209-1216.

Gurtin, M.E., Murdoch, A.I., 1975. A continuum theory of elastic material surfaces. Arch. Ration. Mech. Anal. 57, 291-323.

Hashin, Z., 1962. The elastic moduli of heterogeneous materials. J. Appl. Mech. 29, $143-150$.

Hashin, Z., 1991. Thermoelastic properties of particulate composites with impefect interface. J. Mech. Phys. Solids 39, 745-762.

Hashin, Z., 2001. Thin interphase/imperfect interface in conduction. J. Appl. Phys. 89, $2261-2267$.

Hashin, Z., 2002. Thin interphase/imperfect interface in elasticity with application to coated fiber composites. J. Mech. Phys. Solids 50, 2509-2537.

Hashin, Z., Rosen, B.W., 1964. The elastic moduli of fiber-reinforced materials. J. Appl. Mech. 31, 223-232.

He, J., Lilley, C.M., 2008. Surface effect on the elastic behavior of static bending nanowires. Nano Lett. 8, 1798-1802.

Hill, R., 1963. Elastic properties of reinforced solids: some theoretical principles. J. Mech. Phys. Solids 11, 357-372.

Hill, R., 1972. On constitutive macro-variables for heterogeneous solids at finite strain. Proc. R. Soc. A 326, 131-147.

Huang, Z.P., Sun, L., 2007. Size-dependent effective properties of a heterogeneous material with interface energy effect: from finite deformation theory to infinitesimal strain analysis. Acta Mech. 190, 151-163.

Huang, Z.P., Wang, J., 2006. A theory of hyperelasticity of multi-phase media with surface/interface energy effect. Acta Mech. 182, 195-210.

Javili, A., 2017. Variational formulation of generalized interfaces for finite deformation elasticity. Math. Mech. Solids 23, 303-322.

Javili, A., 2018. A note on traction continuity across an interface in a geometrically nonlinear framework. Math. Mech. Solids. https://doi.org/10.1177/ 1081286518766980

Javili, A., dell'Isola, F., Steinmann, P., 2013. Geometrically nonlinear higher-gradient elasticity with energetic boundaries. J. Mech. Phys. Solids 61, 2381-2401.

Javili, A., Mcbride, A., Mergheim, J., Steinmann, P., Schmidt, U., et al., 2013. Micro-tomacro transitions for continua with surface structure at the microscale. Int. J. Solids Struct. 50, 2561-2572.

Javili, A., McBride, A., Steinmann, P., 2013. Thermomechanics of solids with lower-dimensional energetics: on the importance of surface, interface, and curve structures at the nanoscale. A unifying review. Appl. Mech. Rev. 65, 010802.

Javili, A., Steinmann, P., Mosler, J., 2017. Micro-to-macro transition accounting for general imperfect interfaces. Comput. Methods Appl. Mech. Eng. 317, 274-317.

Kanouté, P., Boso, D.P., Chaboche, J.L., Schrefler, B.A., et al., 2009. Multiscale methods for composites: a review. Arch. Comput. Methods Eng. 16, 31-75.

Khisaeva, Z.F., Ostoja-Starzewski, M., 2006. On the size of RVE in finite elasticity of random composites. J. Elast. 85, 153-173.

Klarbring, A., 1991. Derivation of a model of adhesively bonded joints by the asymptotic expansion method. Int. J. Eng. Sci. 29, 493-512.

Koutsawa, Y., Karatrantos, A., Yu, W., Ruch, D., et al., 2018. A micromechanics approach for the effective thermal conductivity of composite materials with general linear imperfect interfaces. Compos. Struct. . 200, 747-756.

Kushch, V.I., Mogilevskaya, S.G., Stolarski, H.K., Crouch, S.L., et al., 2013. Elastic fields and effective moduli of particulate nanocomposites with the Gurtin-Murdoch model of interfaces. Int. J. Solids Struct. 50, 1141-1153.

Levitas, V.I., Samani, K., 2011. Size and mechanics effects in surface-induced melting of nanoparticles. Nat. Commun. 2, 284-286.

Liu, J.-T., Xie, F.-Y., He, Q.-C., Tang, S.-L., Xiao, C.W., et al., 2019. Effective elastic isotropic moduli of highly filled particulate composites with arbitrarily shaped inhomogeneities. Mech. Mater. 135, 35-45.

Liu, L., Yu, M., Lin, H., Foty, R., et al., 2017. Deformation and relaxation of an incompressible viscoelastic body with surface viscoelasticity. J. Mech. Phys. Solids 98, 309-329. 
Matouš, K., Geers, M.G.D., Kouznetsova, V.G., Gillman, A., et al., 2017. A review of predictive nonlinear theories for multiscale modeling of heterogeneous materials. J. Comput. Phys. 330, 192-220.

Moeckel, G.P., 1975. Thermodynamics of an interface. Arch. Ration. Mech. Anal. 57, 255-280.

Moës, N., Belytschko, T., 2002. Extended finite element method for cohesive crack growth. Eng. Fract. Mech. 69, 813-833.

Mogilevskaya, S.G., Crouch, S.L., Stolarski, H.K., 2008. Multiple interacting circular nanoinhomogeneities with surface/interface effects. J. Mech. Phys. Solids 56, 2298-2327.

Monchiet, V., Bonnet, G., 2010. Interfacial models in viscoplastic composites materials. Int. J. Eng. Sci. 48, 1762-1768.

Monteiro, E., He, Q.C., Yvonnet, J., 2011. Hyperelastic large deformations of two-phase composites with membrane-type interface. Int. J. Eng. Sci. 49, 985-1000.

Murdoch, A.I., 1976. A thermodynamical theory of elastic material interfaces. Q. J. Mech. Appl. Math. 29, 245-275.

Nazarenko, L., Bargmann, S., Stolarski, H., 2017. Closed-form formulas for the effective properties of random particulate nanocomposites with complete Gurtin-Murdoch model of material surfaces. Continuum Mech. Thermodyn. 29, 77-96.

Needleman, A., 1987. A continuum model for void nucleation by inclusion debonding. J. Appl. Mech. 54, 525-531.

Ogden, R.W., 1974. On the overall moduli of non-linear elastic composite materials. J. Mech. Phys. Solids 22, 541-553.

Olsson, P.A.T., Park, H.S., 2012. On the importance of surface elastic contributions to the flexural rigidity of nanowires. J. Mech. Phys. Solids 60, 2064-2083.

Ortiz, M., Pandolfi, A., 1999. Finite-deformation irreversible cohesive elements for threedimensional crack-propagation analysis. Int. J. Numer. Methods Eng. 1282, $1267-1282$

Park, H.S., Klein, P.A., 2008. Surface stress effects on the resonant properties of metal nanowires: the importance of finite deformation kinematics and the impact of the residual surface stress. J. Mech. Phys. Solids 56, 3144-3166.

Park, K., Paulino, G.H., 2011. Cohesive zone models: a critical review of traction-separation relationships across fracture surfaces. Appl. Mech. Rev. 64, 060802

Park, K., Paulino, G.H., Roesler, J.R., 2009. A unified potential-based cohesive model of mixed-mode fracture. J. Mech. Phys. Solids 57, 891-908.

Pham Huy, H., Sanchez-Palencia, E., 1974. Phénomènes de transmission à travers des couches minces de conductivité Élevée. J. Math. Anal. Appl. 47, 284-309.

Pindera, M.J., Khatam, H., Drago, A.S., Bansal, Y., et al., 2009. Micromechanics of spatially uniform heterogeneous media: a critical review and emerging approaches. Compos. Part B 40, 349-378.

Qian, J., Lin, J., Xu, G.K., Lin, Y., Gao, H., et al., 2017. Thermally assisted peeling of an elastic strip in adhesion with a substrate via molecular bonds. J. Mech. Phys. Solids 101, 197-208.

Remmers, J.J.C., de Borst, R., Needleman, A., 2008. The simulation of dynamic crack propagation using the cohesive segments method. J. Mech. Phys. Solids 56, 70-92.

Saeb, S., Steinmann, P., Javili, A., 2016. Aspects of computational homogenization at finite deformations: a unifying review from Reuss' to Voigt's bound. Appl. Mech. Rev. 68, 050801.

Sanchez-Palencia, E., 1970. Comportement limite d'un probléme de transmissioná travers une plaque faiblement conductrice. C. R. Math. Acad. Sci. 270, 1026-1028.

Sevostianov, I., Mogilevskaya, S.G., Kushch, V.I., 2019. Maxwell'S methodology of estimating effective properties: alive and well. Int. J. Eng. Sci. 140, 35-88.

Sharma, P., 2004. Size-dependent elastic fields of embedded inclusions in isotropic chiral solids. Int. J. Solids Struct. 41, 6317-6333.

Sharma, P., Ganti, S., 2004. Size-dependent Eshelby's tensor for embedded nano-inclusions incorporating surface-interface energies. J. Appl. Mech. 71, 663-671.

Sharma, P., Wheeler, L.T., 2007. Size-dependent elastic state of ellipsoidal nano-inclusions incorporating surface-interface tension. J. Appl. Mech. 74, 447-454.

Steigmann, D.J., Ogden, R.W., 1999. Elastic surface substrate interactions. Proc. R. Soc. London A 455, 437-474.

Temizer, I., Zohdi, T.I., 2007. A numerical method for homogenization in non-linear elasticity. Comput. Mech. 40, 281-298.

Tian, L., Rajapakse, R.K.N.D., 2007. Analytical solution for size-dependent elastic field of a nanoscale circular inhomogeneity. J. Appl. Mech. 74, 568-574.

van den Bosch, M.J., Schreurs, P.J.G., Geers, M.G.D., 2006. An improved description of the exponential $\mathrm{Xu}$ and Needleman cohesive zone law for mixed-mode decohesion. Eng. Fract. Mech. 73, 1220-1234.

Wang, G., Chen, Q., He, Z., Pindera, M.J., 2018. Homogenized moduli and local stress fields of unidirectional nano-composites. Compos. Part B 138, 265-277.

Wang, J., Duan, H.L., Zhang, Z., Huang, Z.P., et al., 2005. An anti-interpenetration model and connections between interphase and interface models in particle-reinforced composites. Int. J. Mech. Sci. 47, 701-718.

Wang, Z., Oelkers, R.J., Lee, K.C., Fisher, F.T., et al., 2016. Annular coated inclusion model and applications for polymer nanocomposites part i: spherical inclusions. Mech. Mater. 101, 170-184.

Wang, Z., Oelkers, R.J., Lee, K.C., Fisher, F.T., et al., 2016. Annular coated inclusion model and applications for polymer nanocomposites part ii: cylindrical inclusions. Mech. Mater. 101, 50-60.

Wang, Z.Q., Zhao, Y.P., Huang, Z.P., 2010. The effects of surface tension on the elastic properties of nano structures. Int. J. Eng. Sci. 48, 140-150.

Wells, G.N., Sluys, L.J., 2001. A new method for modelling cohesive cracks using finite elements. Int. J. Numer. Methods Eng. 50, 2667-2682.

Wu, C., Gowrishankar, S., Huang, R., Liechti, K.M., et al., 2016. On determining mixedmode traction-separation relations for interfaces. Int. J. Fract. 202, 1-19.

Yvonnet, J., Quang, H.L., He, Q.C., 2008. An XFEM/level set approach to modelling surface/interface effects and to computing the size-dependent effective properties of nanocomposites. Comput. Mech. 42, 119-131.

Zemlyanova, A.Y., Mogilevskaya, S.G., 2018. On spherical inhomogeneity with Steigmann-Ogden interface. J. Appl. Mech. 85, 121009.

Zhong, Z., Meguid, S.A., 1997. On the elastic field of a spherical inhomogeneity with an imperfectly bonded interface. J. Elast. 46, 91-113. 